Article

\title{
Conjugated Polymer Films Having a Uniaxial Molecular Orientation and Network Structure Prepared by Electrochemical Polymerization in Liquid Crystals
}

\author{
Jiuchao Dong ${ }^{1}$, Shigeki Nimori ${ }^{2}$ and Hiromasa Goto ${ }^{1, *(D)}$ \\ 1 Division of Materials Science, Faculty of Pure and Applied Sciences, University of Tsukuba, \\ Tsukuba 305-8573, Japan; extra9dong@gmail.com \\ 2 National Institute for Materials Science, Tsukuba 305-0003, Japan; nimori.shigeki@nims.go.jp \\ * Correspondence: gotoh@ims.tsukuba.ac.jp
}

check for

updates

Citation: Dong, J.; Nimori, S.; Goto, H. Conjugated Polymer Films Having a Uniaxial Molecular Orientation and Network Structure Prepared by Electrochemical Polymerization in Liquid Crystals. Polymers 2021, 13, 2425. https://doi.org/10.3390/ polym13152425

Academic Editors: Łukasz Klapiszewski and Beata Podkościelna

Received: 21 June 2021

Accepted: 19 July 2021

Published: 23 July 2021

Publisher's Note: MDPI stays neutral with regard to jurisdictional claims in published maps and institutional affiliations.

Copyright: (c) 2021 by the authors. Licensee MDPI, Basel, Switzerland. This article is an open access article distributed under the terms and conditions of the Creative Commons Attribution (CC BY) license (https:// creativecommons.org/licenses/by/ $4.0 /)$.

\begin{abstract}
A new method for fabricating conjugated polymer films was developed using electrochemical polymerization in liquid crystals and magnetic orientation. A uniaxial main chain orientation and a crosslinked network structure were achieved with this method. By employing eight types of monomers, the influence of the crosslinking for the film was investigated. The crosslinking was found to improve the solvent resistance of the conjugated polymer films. This new method is expected to be useful in various applications, such as high-powered organic electronic devices with durability.
\end{abstract}

Keywords: orientation; crosslink; conjugated polymers; electrochemical polymerization; liquid crystal

\section{Introduction}

Due to their unique chemical structure, conjugated polymers can demonstrate inorganic semiconductor-like properties and are thus regarded as a promising material for applications. Many researchers have contributed toward the development of organic electronic devices having conjugated polymers, such as organic field-effect transistors [1-3], organic photovoltaic devices [4,5], and organic light-emitting diodes [6-8]. Compared with inorganic semiconductor materials, conjugated polymers usually have a linear form, which provides anisotropy at the molecular level. The optical absorption, emission properties, and electrical conductivity highly depend on the molecular arrangement [9-12]. Although conjugated polymers can form a well-organized structural arrangement through self-assembly, polymer films generally form poly-domains or a random arrangement. As a result, the electronic devices based on the polymers do not exhibit anisotropic optical or electrical performances. To address this concern, many groups have developed technologies to control the arrangement of conjugated polymers [13-16]. These methods include the synthesis of liquid crystalline polymers [17-20] using mechanical force [21], and oriented substrates to fabricate conjugated polymer films with uniaxial alignment [22,23].

The durability of conjugated polymer films is important for applications in organic electronic devices [24,25]. A possible solution to this issue is to construct a network structure of conjugated polymers by crosslinking; this can help in stabilizing the morphology of the conjugated polymer film [26,27]. However, molecular orientation is somewhat difficult to achieve for polymers with a network structure. A photochemical reaction or a coordination reaction allows the polymers to have both well-organized and crosslinked forms [28,29]. However, there remains a significant technical challenge to apply this technology to other conjugated polymers.

Electrochemical polymerization is a convenient method to synthesize conjugated polymer films. After polymerization, the conjugated polymers usually separate from the electrolyte solution and are then directly deposited on the electrode substrate to form films. Many groups have investigated electrochemical polymerization in terms of its 
mechanism [30-33] and applications, including chemical sensors [34,35], electrochromic materials [36], electroluminescent materials [37,38], and organic photovoltaic devices [39]. Our group developed a new electrochemical polymerization in a liquid crystal (LC) electrolyte solution. In contrast to traditional isotropic solvents, when LC solvents are used as the reaction medium for electrochemical polymerization, the resultant conjugated polymers tend to align along the director of the liquid crystals (LCs). The polymer films prepared in the LC have a molecular order, which is similar to that of the LCs. When smectic A LCs were employed, polarized optical microscopy (POM) observations indicated that the resulting conjugated polymer films showed a fan-shaped birefringence texture, which is the typical optical texture of smectic A LCs [40]. When cholesteric LCs were employed for the polymerization, the resulting films had an intermolecular helical order and showed a fingerprint texture similar to that of cholesteric LCs with circular dichroism optical absorption [41-44]. The most significant aspect of this preparation method is the alignment of the conjugated polymers, which can be modified by adjusting the orientation of the host LC molecules. Conjugated polymer films having a unidirectionally oriented molecular alignment were fabricated using a magnetic field [45]. We found a new way to fabricate conjugated polymer films whose molecular orientation can be controlled with a magnetic field in the polymerization process.

In this study, using electrochemical polymerization in LCs, a new method was developed to fabricate conjugated polymer films having a uniaxial molecular orientation and a crosslinked network structure. Both the uniaxial molecular orientation and preparation of crosslinked network structures allow the conjugated polymer films to show anisotropic optical properties and durability, which may endow organic electronic devices with high performance. To investigate the influence of crosslinking, eight types of monomers were employed in the synthesis for the production of crosslinked and uncrosslinked polymers. Electrochemical polymerization was conducted in a $12 \mathrm{~T}$ magnetic field generated by a superconducting magnet to ensure occurrence of the polymerization in an LC reaction medium with orientation. The resulting conjugated polymer films were characterized by POM observations and linear dichroism (LD) UV-vis spectroscopy to investigate the following issues: (1) uniaxial orientation, (2) orientation degree of the polymers, and (3) optical properties of the films.

\section{Experimental}

\subsection{Materials}

Eight types of monomer were used, including 2,2'-bithiophene (BT), 1,6-di(2,2'-bithiophen3-yl)hexane (d(BT)), 9-butyl-2,7-di(thiophen-2-yl)-9H-carbazole (DTC), 1,6-bis(2,7-di(thiophen2-yl)-9H-carbazol-9-yl)hexane (bis(DTC)), 1,4-dimethoxy-2,5-di(thiophen-2-yl)benzene (DTB), 1,6-bis(4-methoxy-2,5-di(thiophen-2-yl)phenoxy)hexane (bis(DTB)), 1-ethoxy-4-methoxy-2,5di(thiophen-2-yl)benzene (DTB2), and 1-butoxy-4-methoxy-2,5-di(thiophen-2-yl)benzene (DTB4), and they are shown in Scheme 1. In addition, the matrix LC 4-cyano-4'-hexylbiphenyl (6CB) and the supporting salt tetrabutylammonium perchlorate (TBAP) are shown in Scheme 1. The syntheses of the monomers d(BT), bis(DTC), DTB, bis(DTB), DTB2, and DTB4 are illustrated in Scheme 2. The synthesis of DTC was reported in our previous work [46]. The monomer BT and the supporting salt TBAP were purchased from Tokyo Chemical Industry (TCI). The LC 6CB was purchased from Merck. All other necessary chemicals were purchased from TCI, Wako Pure Chemical, Nacalai Tesque, and Sigma-Aldrich.

\subsection{Synthesis}

\subsubsection{Trimethyl(thiophen-2-yl)stannane (1)}

First, 2-bromothiophene $(1.63 \mathrm{~g}, 10 \mathrm{mmol})$ was dissolved in dry tetrahydrofuran (THF, $35 \mathrm{~mL}$ ) and cooled to $-78{ }^{\circ} \mathrm{C}$. A solution of $n$-butyllithium in hexane $(6.88 \mathrm{~mL}$, $11 \mathrm{mmol}$ ) was added dropwise to the mixture and stirred for $1.5 \mathrm{~h}$ at $-78{ }^{\circ} \mathrm{C}$. Then, trimethyltinchloride $(2.39 \mathrm{~g}, 12 \mathrm{mmol})$ was added to the solution at $-78^{\circ} \mathrm{C}$ and the mixture 


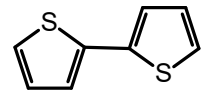

BT

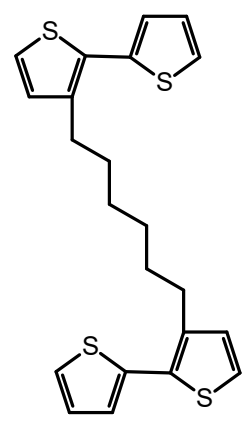

d(BT)

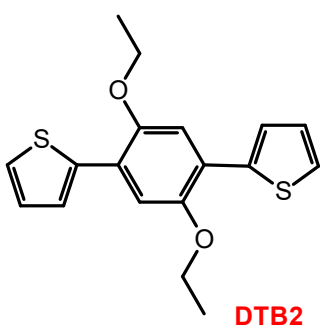

was allowed to slowly warm to room temperature followed by stirring for $24 \mathrm{~h}$. Then, dichloromethane (DCM) was used to extract the product. The combined organic extracts were dried using $\mathrm{Na}_{2} \mathrm{SO}_{4}$, followed by filtration and evaporation to yield $\mathbf{1}$ as a yellow liquid $(2.16 \mathrm{~g}, 8.75 \mathrm{mmol}, 87.5 \%)$.

${ }^{1} \mathrm{H}$ NMR (400 MHz; $\left.\mathrm{CDCl}_{3} ; \mathrm{TMS}\right) \delta(\mathrm{ppm}) 0.376(\mathrm{~s}, 9 \mathrm{H}), 7.223-7.273(\mathrm{~m}, 2 \mathrm{H}), 7.649(\mathrm{~d}$, $1 \mathrm{H}, J=5.6 \mathrm{~Hz}) .{ }^{13} \mathrm{C} \mathrm{NMR}\left(100 \mathrm{MHz} ; \mathrm{CDCl}_{3}\right.$; TMS) $\delta$ (ppm) 127.895, 130.716, 134.948137 .064 .

\subsubsection{4,4,5,5-Tetramethyl-2-(thiophen-2-yl)-1,3,2-dioxaborolane (2)}

2-Bromothiophene (1.63 g, $10 \mathrm{mmol})$ was dissolved in dry THF (35 mL) and cooled down to $-78^{\circ} \mathrm{C}$. A solution of $n$-butyllithium in hexanes $(6.56 \mathrm{~mL}, 10.5 \mathrm{mmol})$ was added dropwise, and the mixture was stirred for $2 \mathrm{~h}$ at $-78^{\circ} \mathrm{C}$. 2-Isopropoxy-4,4,5,5-tetramethyl1,3,2-dioxaborolane $(2.05 \mathrm{~g}, 11 \mathrm{mmol})$ was subsequently added at $-78^{\circ} \mathrm{C}$, and the mixture was allowed to slowly warm up to room temperature and stirred for $22 \mathrm{~h}$. After the reaction, the mixture was extracted with DCM. The combined organic extracts were dried by $\mathrm{MgSO}_{4}$, followed by filtration, and was purified by silica gel column chromatography (eluent: hexane/ethyl acetate $=9 / 1)$ to yield a yellow liquid $(1.04 \mathrm{~g}, 4.96 \mathrm{mmol}, \mathrm{Y}=49.6 \%)$.

${ }^{1} \mathrm{H} \mathrm{NMR}\left(400 \mathrm{MHz} ; \mathrm{CDCl}_{3}\right.$; TMS) $\delta(\mathrm{ppm}) 1.278(\mathrm{~s}, 12 \mathrm{H}), 7.108-7.128(\mathrm{dd}, 1 \mathrm{H}, J=3.2$, $4.4 \mathrm{~Hz}), 7.552-7.576(\mathrm{dd}, 1 \mathrm{H}, J=1.2,8.8 \mathrm{~Hz}), 7.566-7.581(\mathrm{~d}, 1 \mathrm{H}, J=6.0 \mathrm{~Hz}) .{ }^{13} \mathrm{C} \mathrm{NMR}$ $\left(100 \mathrm{MHz} ; \mathrm{CDCl}_{3}\right.$; TMS) $\delta$ (ppm) 24.828, 84.081, 115.905, 128.210, 132.356, 137.140.
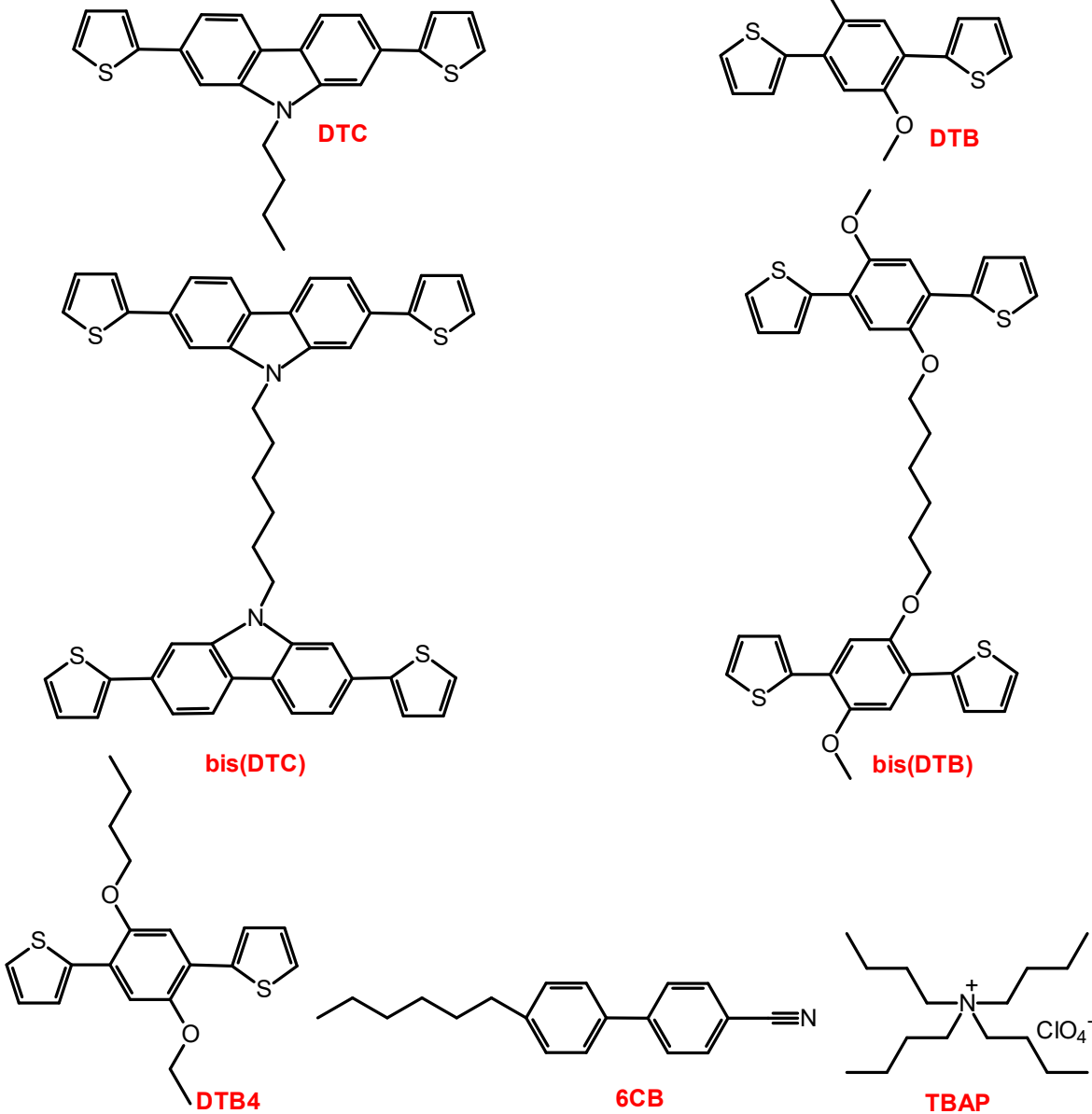

TBAP

Scheme 1. Monomers employed for the electrochemical polymerization in liquid crystals, and molecular structures of liquid crystal 4-cyano-4'-hexylbiphenyl (6CB) and the supporting salt tetrabutylammonium perchlorate (TBAP). 


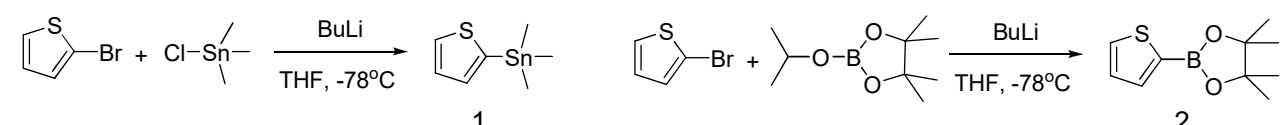

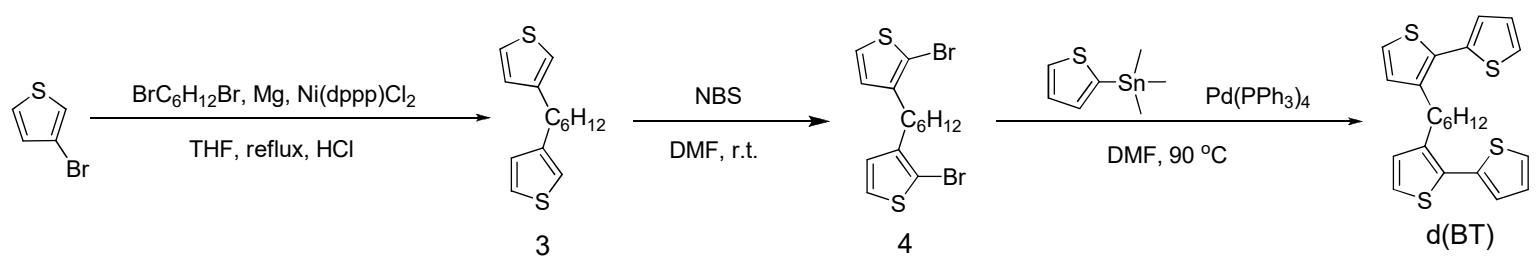<smiles>O=[N+]([O-])c1cc(Br)ccc1-c1ccc(Br)cc1</smiles><smiles>CC[Si](C)(CC(C)C)c1cccs1</smiles>

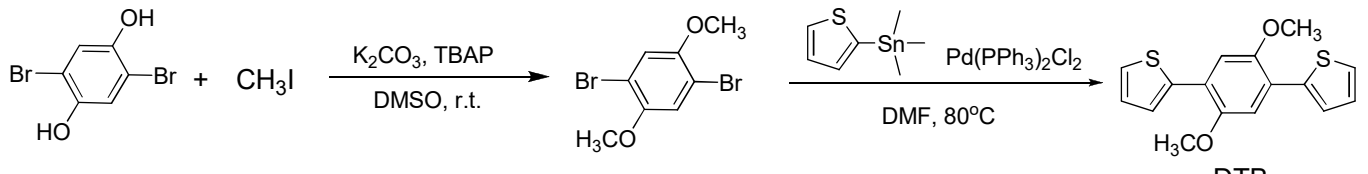

8

DTB

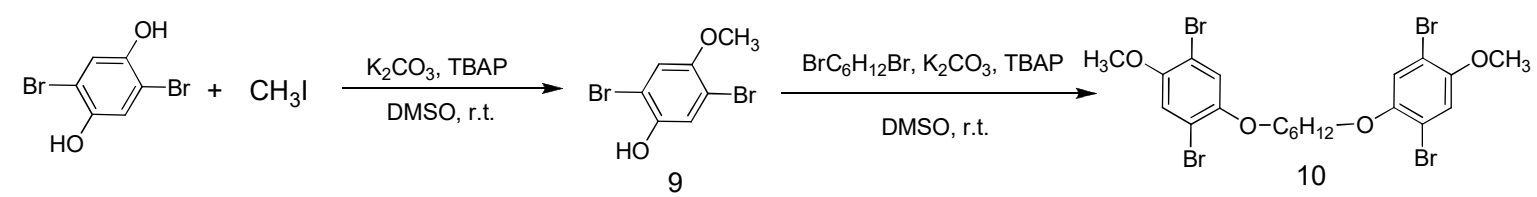<smiles>CCOc1cc(-c2cccs2)c(OC)cc1-c1cccs1</smiles>

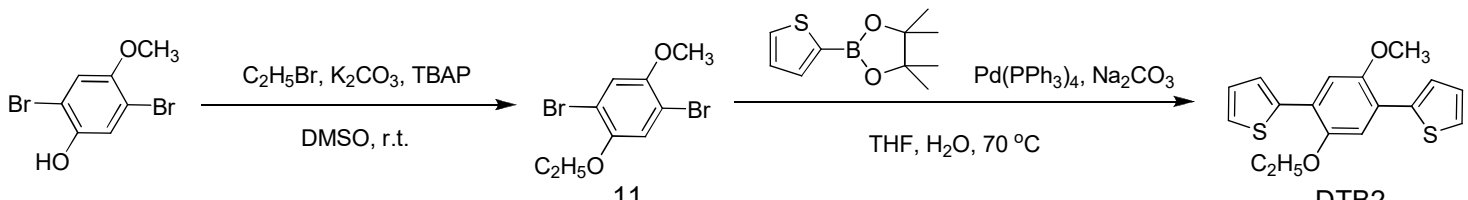

11

DTB2

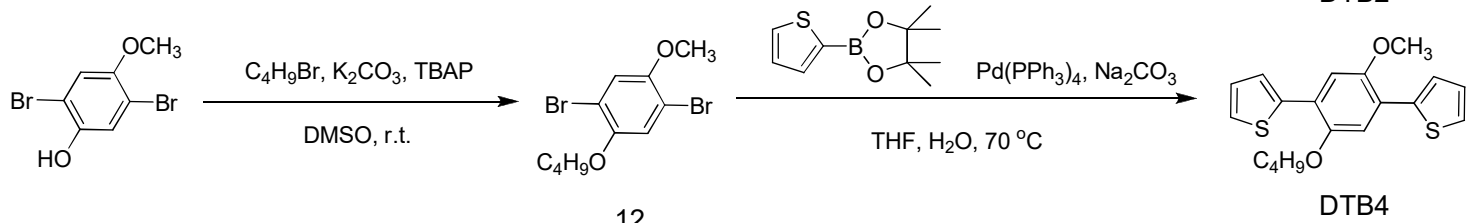

Scheme 2. Synthesis for monomers d(BT), bis(DTC), DTB, bis(DTB), DTB2, and DTB4.

\subsubsection{1,6-Di(thiophen-3-yl)hexane (3)}

1,6-Bromohexane $(2.44 \mathrm{~g}, 10 \mathrm{mmol})$ was slowly added to a suspension of magnesium turnings $(0.583 \mathrm{~g}, 24 \mathrm{mmol})$ in $25 \mathrm{~mL}$ of anhydrous THF and a spot of iodine followed by reflux for $3 \mathrm{~h}$. 3-Bromothiophene (3.42 g, $21 \mathrm{mmol}$ ) and dichloro[1,3-bis(diphenylphosphino) 
propane]nickel $(0.054 \mathrm{~g}, 0.1 \mathrm{mmol})$ was dissolved in $20 \mathrm{~mL}$ of THF. Then the Grignard solution was transferred via injection syringe and added dropwise to this solution at $0{ }^{\circ} \mathrm{C}$. The resulting mixture was stirred at room temperature for $15 \mathrm{~h}$, hydrolyzed with $1 \mathrm{M}$ of $\mathrm{HCl}(14 \mathrm{~mL})$ at $0{ }^{\circ} \mathrm{C}$, followed by extraction with DCM. The combined organic extracts were dried over $\mathrm{MgSO}_{4}$ and purified by silica gel column chromatography (eluent: hexane), followed by drying, to give a colorless liquid $(2.5 \mathrm{~g}, 10 \mathrm{mmol}, \mathrm{Y}=100 \%)$.

${ }^{1} \mathrm{H}$ NMR (400 MHz; $\mathrm{CDCl}_{3}$; TMS) $\delta$ (ppm) 1.342 (quint, $4 \mathrm{H}, J=3.8 \mathrm{~Hz}$ ), 1.583 (quint, $4 \mathrm{H}, J=7.5 \mathrm{~Hz}), 2.595(\mathrm{t}, 4 \mathrm{H}, J=7.6), 6.927-6.896(\mathrm{~m}, 4 \mathrm{H}), 7.215-7.236(\mathrm{dd}, 2 \mathrm{H}, J=3.2$, $5.2 \mathrm{~Hz}) .{ }^{13} \mathrm{C}$ NMR (100 MHz; CDCl 3 ; TMS) $\delta(\mathrm{ppm}) 29.069,30.241,30.479,119.784,125.017$, $128.229,143.088$.

\subsubsection{1,6-Bis(2-bromothiophen-3-yl)hexane (4)}

To a solution of 1,6-di(thiophen-3-yl)hexane $(2.50 \mathrm{~g}, 10 \mathrm{mmol})$ in dimethylformamide (DMF) $(20 \mathrm{~mL}), N$-bromosuccinimide $(4.9 \mathrm{~g}, 23 \mathrm{mmol})$ was slowly added at room temperature. The mixture was stirred at room temperature for 2 days. After the reaction, the mixture was extracted with ether and dried over $\mathrm{MgSO}_{4}$, followed by purification by silica gel column chromatography (eluent: hexane) to yield 4 as a yellow oil $(1.274 \mathrm{~g}, 3.12 \mathrm{mmol}$, $\mathrm{Y}=31.2 \%)$.

${ }^{1} \mathrm{H}$ NMR (400 MHz; $\mathrm{CDCl}_{3}$; TMS) $\delta$ (ppm) 1.341 (quint, $4 \mathrm{H}, J=3.7 \mathrm{~Hz}$ ), 1.538 (quint, $4 \mathrm{H}, J=7.3 \mathrm{~Hz}), 2.536(\mathrm{t}, 4 \mathrm{H}, J=7.6 \mathrm{~Hz}), 6.768(\mathrm{~d}, 2 \mathrm{H}, J=5.2 \mathrm{~Hz}), 7.166(\mathrm{~d}, 2 \mathrm{H}$, $J=6.0 \mathrm{~Hz}) .{ }^{13} \mathrm{C}$ NMR $\left(100 \mathrm{MHz} ; \mathrm{CDCl}_{3}\right.$; TMS) $\delta$ (ppm) 28.907, 29.345, 29.641, 108.862, $125.179,128.191,141.782$.

\subsubsection{1,6-Di(2,2'-bithiophen-3-yl)hexane (d(BT))}

Under an argon atmosphere, 1,6-bis(2-bromothiphen-3-yl)hexane $(0.163 \mathrm{~g}, 0.4 \mathrm{mmol})$ and trimethyl(thiophen-2-yl)stannane $(0.247,1 \mathrm{mmol})$ were weighed into a two-neck flask and dissolved in $2 \mathrm{~mL}$ of DMF, followed by degassing for $10 \mathrm{~min}$. Then, tetrakis (triphenylphosphine)palladium(0) $(0.034 \mathrm{~g}, 0.03 \mathrm{mmol})$ was added. After stirring at $90{ }^{\circ} \mathrm{C}$ for $22 \mathrm{~h}$, followed by cooling down, the solution was extracted with chloroform and dried over $\mathrm{MgSO}_{4}$. The product was purified by silica gel column chromatography (eluent: hexane/ethyl acetate $=47 / 3)$ to yield $\mathbf{d}(\mathbf{B T})$ as a green oil $(0.122 \mathrm{~g}, 0.29 \mathrm{mmol}, \mathrm{Y}=73 \%)$.

${ }^{1} \mathrm{H}$ NMR (400Hz; DMSO; TMS) $\delta(\mathrm{ppm}) 1.345$ (quint, $4 \mathrm{H}, J=3.5 \mathrm{~Hz}$ ), 1.576 (quint, $4 \mathrm{H}$, $J=7.3 \mathrm{~Hz}), 2.701(\mathrm{t}, 4 \mathrm{H}, J=8.0 \mathrm{~Hz}), 6.897(\mathrm{~d}, 2 \mathrm{H}, J=5.6 \mathrm{~Hz}), 7.030(\mathrm{~m}, 2 \mathrm{H}), 7.080(\mathrm{~d}, 2 \mathrm{H}$, $J=3.6 \mathrm{~Hz}), 7.149(\mathrm{~d}, 2 \mathrm{H}, J=5.6 \mathrm{~Hz}), 7.272(\mathrm{~d}, 2 \mathrm{H}, J=5.2 \mathrm{~Hz}) .{ }^{13} \mathrm{C}$ NMR $(100 \mathrm{~Hz} ; \mathrm{DMSO}$; TMS) $\delta$ (ppm) 29.021, 30.327, 34.235, 123.730, 125.274, 125.493, 125.999, 127.285, 129.840, 136.140, 139.504 .

\subsubsection{4,4'-Dibromo-2-nitro-biphenyl (5)}

Under an argon atmosphere, 4,4'-dibromobiphenyl ( $3.12 \mathrm{~g}, 10 \mathrm{mmol})$ was weighed into a two-neck flask and dissolved in $10 \mathrm{~mL}$ of DCM and $30 \mathrm{~mL}$ of acetic anhydride. Then, $5 \mathrm{~mL}$ of acetic acid and $9.3 \mathrm{~mL}$ of nitric acid were added dropwise with an ice bath. After addition, the mixture was allowed to warm up to room temperature. After stirring for $3 \mathrm{~h}$, the solution was extracted with DCM and dried over $\mathrm{MgSO}_{4}$. The solvent was removed on a rotary evaporator, and the residue was purified by silica gel column chromatography, (eluent: hexane/ethyl acetate $=4 / 1$ ), followed by evaporation, to yield 5 as a yellow solid (1.84 g, $5.15 \mathrm{mmol}, \mathrm{Y}=51 \%)$.

${ }^{1} \mathrm{H}$ NMR $\left(400 \mathrm{~Hz} ; \mathrm{CDCl}_{3}\right.$; TMS) $\delta(\mathrm{ppm}) 7.142-7.160(\mathrm{~d}, 2 \mathrm{H}, J=7.2 \mathrm{~Hz}), 7.272-7.293$ $(\mathrm{d}, 1 \mathrm{H}, J=8.4 \mathrm{~Hz}), 7.547-7.568(\mathrm{~d}, 2 \mathrm{H}, J=8.4 \mathrm{~Hz}), 7.736-7.763(\mathrm{dd}, 1 \mathrm{H}, J=8.0,2.8 \mathrm{~Hz})$, $8.022-8.027(\mathrm{~d}, 1 \mathrm{H}, J=2.0 \mathrm{~Hz}) .{ }^{13} \mathrm{C}$ NMR $\left(100 \mathrm{~Hz} ; \mathrm{CDCl}_{3}\right.$; TMS) $\delta$ (ppm) 100.179, 121.862, $123.006,127.323,129.439,131.994,132.966,134.205,135.434,163.817,172,624$.

\subsubsection{2,7-Dibromo-9H-carbazole (6)}

Under an argon atmosphere, 4,4'-dibromo-2-nitro-biphenyl (1.84 g, $5.15 \mathrm{mmol})$ and triphenylphosphine $(4.05 \mathrm{~g}, 15.5 \mathrm{mmol})$ were weighed into a two-neck flask and dissolved 
with $15 \mathrm{~mL}$ of $o$-dichlorobenzene. The mixture was heated to $180^{\circ} \mathrm{C}$. After stirring for $19 \mathrm{~h}$, the solvent was removed with a rotary evaporator, and the residue was purified by silica gel (eluent: hexane/chloroform $=3 / 1$ ) followed by evaporation to yield $\mathbf{6}$ as a brown solid (1.29 $\mathrm{g}, 3,97 \mathrm{mmol}, \mathrm{Y}=77 \%)$.

${ }^{1} \mathrm{H} \mathrm{NMR}\left(400 \mathrm{~Hz} ; \mathrm{CDCl}_{3}\right.$; TMS) $\delta(\mathrm{ppm}) 7.336-7.360(\mathrm{dd}, 2 \mathrm{H}, J=8.0,1.2 \mathrm{~Hz}), 7.563-7.568$ $(\mathrm{d}, 2 \mathrm{H}, \mathrm{J}=2.0 \mathrm{~Hz}), 7.856-7.877(\mathrm{~d}, 2 \mathrm{H}, \mathrm{J}=8.4 \mathrm{~Hz}), 8.056(\mathrm{~s}, 1 \mathrm{H}) .{ }^{13} \mathrm{C} \mathrm{NMR}\left(100 \mathrm{~Hz} ; \mathrm{CDCl}_{3}\right.$; TMS) $\delta(\mathrm{ppm}) 113.789,119.708,121.443,121.738,123.263,140.228$.

\subsubsection{1,6-Bis(2,7-dibromo-9H-carbazol-9yl)hexane (7)}

Under an argon atmosphere, 2,7-dibromo-9H-carbazole (0.325 g, $1 \mathrm{mmol}), 1,6$ dibromohexane $(0.122 \mathrm{~g}, 0.5 \mathrm{mmol})$, sodium hydroxide $(0.08 \mathrm{~g}, 2 \mathrm{mmol})$, and tetrabutylammonium perchlorate $(0.014 \mathrm{~g}, 0.04 \mathrm{mmol})$ were weighed into a two-neck flask and dissolved in $3 \mathrm{~mL}$ of 2-butanone. The mixture was heated to $70{ }^{\circ} \mathrm{C}$. After stirring for $39 \mathrm{~h}$, the organic layer was extracted with chloroform, and then dried by $\mathrm{MgSO}_{4}$ followed by filtration. After evaporation, the residue was collected by recrystallization in chloroform to yield 7 as a brown solid $(0.292 \mathrm{~g}, 0.4 \mathrm{mmol}, \mathrm{Y}=80 \%)$.

${ }^{1} \mathrm{H}$ NMR (400Hz; $\mathrm{CDCl}_{3}$; TMS) $\delta(\mathrm{ppm}) 1.429(\mathrm{~m}, 4 \mathrm{H}), 1.825(\mathrm{~m}, 4 \mathrm{H}), 4.144-4.179(\mathrm{t}$, $4 \mathrm{H}, J=7.0 \mathrm{~Hz}), 7.304-7.325(\mathrm{~d}, 4 \mathrm{H}, J=8.4 \mathrm{~Hz}), 7.468(\mathrm{~s}, 4 \mathrm{H}), 7.841-7.863(\mathrm{~d}, 4 \mathrm{H}, J=8.8 \mathrm{~Hz})$.

\subsubsection{1,6-Bis(2,7-di(thiophen-2-yl)-9H-carbazol-9-yl)hexane (bis(DTC))}

Under an argon atmosphere, 1,6-bis(2,7-dibromo-9H-carbazol-9-yl)hexane (0.146 g, $0.2 \mathrm{mmol}$ ) and trimethyl(thiophen-2-yl)stannane $(0.247,1 \mathrm{mmol})$ were weighed into a twoneck flask and dissolved in $2 \mathrm{~mL}$ of toluene and $2 \mathrm{~mL}$ of THF, followed by degassing for $10 \mathrm{~min}$. Then, tetrakis(triphenylphosphine)palladium(0) $(0.034 \mathrm{~g}, 0.03 \mathrm{mmol})$ was added. After stirring at $90{ }^{\circ} \mathrm{C}$ for 4 days, followed by cooling down, the solution was extracted with chloroform and dried over $\mathrm{MgSO}_{4}$. The product was purified by recrystallization in chloroform/methanol, and column chromatography (hexane/chloroform =2/1) was conducted to yield bis(DTC) as a green solid (0.032 $\mathrm{g}, 0.045 \mathrm{mmol}, \mathrm{Y}=22.5 \%)$.

${ }^{1} \mathrm{H}$ NMR (400Hz; DMSO; TMS) $\delta(\mathrm{ppm}) 1.514(\mathrm{~m}, 4 \mathrm{H}), 1.904(\mathrm{~m}, 4 \mathrm{H}), 4.367-4.401(\mathrm{t}$, $4 \mathrm{H}, J=6.8 \mathrm{~Hz}), 7.078-7.098(\mathrm{~m}, 4 \mathrm{H}), 7.306-7.319(\mathrm{~d}, 4 \mathrm{H}, J=5.2 \mathrm{~Hz}), 7.420-7.458(\mathrm{~m}, 8 \mathrm{H})$, $7.607(\mathrm{~s}, 4 \mathrm{H}), 8.001-8.022(\mathrm{~d}, 4 \mathrm{H}, J=8.4 \mathrm{~Hz})$.

\subsubsection{1,4-Dibromo-2,5-bis-methoxybenzene (8)}

2,5-Dibromohydroquinone $(2.679 \mathrm{~g}, 10 \mathrm{mmol})$, tetrabutylammonium perchlorate $(0.171 \mathrm{~g}, 0.5 \mathrm{mmol})$, and potassium carbonate $(4.15 \mathrm{~g}, 30 \mathrm{mmol})$ were mixed in acetone $(30 \mathrm{~mL})$. Iodomethane $(4.26 \mathrm{~g}, 30 \mathrm{mmol})$ was added to the solution. Then, the mixture was stirred at room temperature for $24 \mathrm{~h}$. The mixture was extracted with DCM twice, and the organic layer was dried over $\mathrm{MgSO}_{4}$. Purification with silica gel column chromatography (eluent: chloroform) gave 8 as a white solid (0.949 $\mathrm{g}, 34 \mathrm{mmol}, \mathrm{Y}=34 \%$ ).

${ }^{1} \mathrm{H}$ NMR (600 MHz; $\mathrm{CDCl}_{3}$; TMS) $\delta$ (ppm) 3.851 (s, 6H), 7.105 (s, 2H).

\subsubsection{1,4-Dimethoxy-2,5-di(thiophen-2-yl)benzene (DTB)}

Under an argon atmosphere, 1,4-dibromo-2,5-dimethoxybenzene (0.296 g, $1 \mathrm{mmol})$ and trimethyl(thiophen-2-yl)stannane $(0.519,2 \mathrm{mmol})$ were weighed into a two-neck flask and dissolved in $5 \mathrm{~mL}$ of DMF, followed by degassing for $10 \mathrm{~min}$. Then, bis(triphenylphosphine) palladium(II)dichloride $(0.048 \mathrm{~g}, 0.12 \mathrm{mmol})$ was added. After stirring at $80^{\circ} \mathrm{C}$ for $24 \mathrm{~h}$, followed by cooling down, the solution was extracted with DCM and dried over $\mathrm{MgSO}_{4}$. The solvent was removed with a rotary evaporator and the residue was purified by silica gel column chromatography (eluent: hexane/chloroform $=2.5 / 1$ ), followed by evaporation, to yield DTB as a green solid $(0.121 \mathrm{~g}, 0.4 \mathrm{mmol}, \mathrm{Y}=40 \%)$.

${ }^{1} \mathrm{H}$ NMR $\left(400 \mathrm{~Hz} ; \mathrm{CDCl}_{3} ; \mathrm{TMS}\right) \delta(\mathrm{ppm}) 3.941(\mathrm{~s}, 6 \mathrm{H}), 7.089-7.111(\mathrm{dd}, 2 \mathrm{H}, \mathrm{J}=5.2$, $3.2 \mathrm{~Hz}), 7.247(\mathrm{~s}, 2 \mathrm{H}), 7.333-7.348(\mathrm{dd}, 2 \mathrm{H}, J=5.2,1.6 \mathrm{~Hz}), 7.520-7.532(\mathrm{dd}, 2 \mathrm{H}, J=3.6$, $2.4 \mathrm{~Hz}) .{ }^{13} \mathrm{C}$ NMR $\left(100 \mathrm{~Hz} ; \mathrm{CDCl}_{3}\right.$; TMS) $\delta$ (ppm) 56.442, 112.331, 122.987, 125.474, 125.694, $126.904,139.037,149.831$. 


\subsubsection{2,5-Dibromo-4-methoxyphenol (9)}

2,5-Dibromohydroquinone ( $2.14 \mathrm{~g}, 8 \mathrm{mmol})$, tetrabutylammonium perchlorate $(0.034 \mathrm{~g}$, $0.1 \mathrm{mmol})$, and potassium carbonate $(1.38 \mathrm{~g}, 10 \mathrm{mmol})$ were mixed in dimethyl sulfoxide (DMSO, $15 \mathrm{~mL}$ ). Iodomethane $(1.135 \mathrm{~g}, 8 \mathrm{mmol})$ was added to the solution dropwise. Then, the mixture was stirred at room temperature for $16 \mathrm{~h}$. The reaction mixture was extracted with DCM and dried over magnesium sulfate. Purification by silica gel column chromatography (eluent: hexane/ethyl acetate $=4 / 1)$ gave 9 as a white solid $(0.784 \mathrm{~g}$, $2.78 \mathrm{mmol}, \mathrm{Y}=35 \%)$.

${ }^{1} \mathrm{H}$ NMR (400 MHz; CDCl $;$ TMS) $\delta$ (ppm) 3.836 (s, 3H), 5.146 (s, 1H), 6.979 (s, 1H), 7.247 (s, $1 \mathrm{H}) .{ }^{13} \mathrm{C}$ NMR (100 MHz; CDCl 3 ; TMS) $\delta$ (ppm) 57.128, 108.376, 111.817, 115.181, $120.413,146.824,150.341$.

\subsubsection{1,6-Bis(2,5-dibromo-4-methoxyphenoxy)hexane (10)}

2,5-Dibromo-4-methoxyphenol (0.564 g, $2 \mathrm{mmol})$, tetrabutylammonium perchlorate $(0.0085 \mathrm{~g}, 0.025 \mathrm{mmol})$, and potassium carbonate $(0.345 \mathrm{~g}, 2.5 \mathrm{mmol})$ were mixed in DMSO $(8 \mathrm{~mL})$. 1,6-Dibromohexane $(0.232 \mathrm{~g}, 0.95 \mathrm{mmol})$ was added to the solution dropwise. Then, the mixture was stirred at room temperature for 3 days. The mixture was extracted with DCM and dried over magnesium sulfate. Purification by silica gel column chromatography (eluent: hexane/ethyl acetate $=9 / 1)$ gave 10 as a pink solid $(0.414 \mathrm{~g}, 0.637 \mathrm{mmol}, \mathrm{Y}=64 \%)$.

${ }^{1} \mathrm{H}$ NMR (400 MHz; $\mathrm{CDCl}_{3}$; TMS) $\delta(\mathrm{ppm}) 1.570$ (quint, $4 \mathrm{H}, J=3.6 \mathrm{~Hz}$ ), 1.817 (quint, $4 \mathrm{H}, J=6.5 \mathrm{~Hz}), 3.840(\mathrm{~s}, 6 \mathrm{H}), 3.956(\mathrm{t}, 4 \mathrm{H}, J=6.2 \mathrm{~Hz}), 7.076(\mathrm{~s}, 2 \mathrm{H}), 7.088(\mathrm{~s}, 2 \mathrm{H}) .{ }^{13} \mathrm{C} \mathrm{NMR}$ $\left(100 \mathrm{MHz} ; \mathrm{CDCl}_{3}\right.$; TMS) $\delta$ (ppm) 25.475, 28.964, 57.023, 69.976, 110.349, 111.187, 116.925, $118.517,150.017,150.426$.

\subsubsection{1,6-Bis(4-methoxy-2,5-di(thiophen-2-yl)phenoxy)hexane (bis(DTB))}

In an argon-flushed two-neck round-bottom flask, 4,4,5,5-tetramethyl-2-(thiophen-2yl)1,3,2-dioxaborolane ( $0.21 \mathrm{~g}, 1 \mathrm{mmol}$ ) and 1,6-bis(2,5-dibromo-4-methoxyphenoxy)hexane $(0.155 \mathrm{~g}, 0.24 \mathrm{mmol})$ were dissolved in THF $(3 \mathrm{~mL})$ and degassed for $10 \mathrm{~min}$. Then, tetrakis(triphenylphosphine)palladium $(0.069 \mathrm{~g}, 0.06 \mathrm{mmol})$ and $2 \mathrm{~mL}$ of the degassed $\mathrm{Na}_{2} \mathrm{CO}_{3}(0.318 \mathrm{~g}, 3 \mathrm{mmol}) /$ water solution were added. The mixture was heated at $70{ }^{\circ} \mathrm{C}$ and refluxed for $24 \mathrm{~h}$. After cooling down, the organic layer was extracted with DCM and dried over $\mathrm{MgSO}_{4}$. The solvent was removed with a rotary evaporator, and the residue was purified by column chromatography (eluent: hexane/ethyl acetate/chloroform $=4 / 1 / 1.5$ ) to yield bis(DTB) as a yellow solid $(0.111 \mathrm{~g}, 0.169 \mathrm{mmol}, \mathrm{Y}=70 \%)$.

${ }^{1} \mathrm{H}$ NMR $\left(400 \mathrm{~Hz} ; \mathrm{CDCl}_{3}\right.$; TMS) $\delta(\mathrm{ppm}) 1.624$ (quint, $4 \mathrm{H}, J=3.8 \mathrm{~Hz}$ ), 1.916 (quint, $4 \mathrm{H}$, $J=6.6 \mathrm{~Hz}), 3.935(\mathrm{~s}, 6 \mathrm{H}), 4.081(\mathrm{t}, 4 \mathrm{H}, J=6.4 \mathrm{~Hz}), 7.054(\mathrm{t}, 2 \mathrm{H}, J=7.0 \mathrm{~Hz}), 7.063(\mathrm{dd}, 2 \mathrm{H}$, $J=1.2,8.8 \mathrm{~Hz}), 7.222(\mathrm{~s}, 2 \mathrm{H}), 7.248(\mathrm{~s}, 2 \mathrm{H}), 7.286-7.335(\mathrm{~m}, 4 \mathrm{H}), 7.498-7.528(\mathrm{~m}, 4 \mathrm{H}) .{ }^{13} \mathrm{C}$ NMR (100Hz; $\left.\mathrm{CDCl}_{3} \mathrm{TMS}\right) \delta(\mathrm{ppm}) 25.990,29.288,56.423,69.442,112.064,112.941,122.901$, $122.987,125.150,125.417,125.636,125.770,126.685,126.856,139.085,149.283,149.788$.

\subsubsection{1,4-Dibromo-2-ethoxy-5-methoxybenzene (11)}

2,5-Dibromo-4-methoxyphenol $(0.226 \mathrm{~g}, 0.8 \mathrm{mmol})$, tetrabutylammonium perchlorate $(0.01 \mathrm{~g}, 0.03 \mathrm{mmol})$, and potassium carbonate $(0.138 \mathrm{~g}, 1 \mathrm{mmol})$ were mixed in DMSO $(3 \mathrm{~mL})$. Bromoethane $(0.075 \mathrm{~mL}, 1 \mathrm{mmol})$ was added dropwise. Then, the mixture was stirred at room temperature for $24 \mathrm{~h}$. The mixture was extracted with DCM and dried over $\mathrm{MgSO}_{4}$. Purification by silica gel column chromatography (eluent: hexane/ethyl acetate $=4 / 1)$ gave 11 as a white solid $(0.25 \mathrm{~g}, 0.8 \mathrm{mmol}, \mathrm{Y}=100 \%)$.

${ }^{1} \mathrm{H}$ NMR (400 MHz; CDCl ; TMS) $\delta(\mathrm{ppm}) 1.425-1.460(\mathrm{t}, 3 \mathrm{H}, J=8.2 \mathrm{~Hz}), 3.844(\mathrm{~s}, 3 \mathrm{H})$, $4.010-4.062(\mathrm{q}, 2 \mathrm{H}, J=6.9 \mathrm{~Hz}), 7.092(\mathrm{~s}, 1 \mathrm{H}), 7.102(\mathrm{~s}, 1 \mathrm{H}) .{ }^{13} \mathrm{C} \mathrm{NMR}\left(100 \mathrm{MHz} ; \mathrm{CDCl}_{3}\right.$; TMS) $\delta(\mathrm{ppm}) 14.763,57.004,66.011,110.387,111.321,116.982,118.812,149.950,150.541$.

\subsubsection{2,2'-(2-Ethoxy-5-methoxy-1,4-phenylene)dithiophene (DTB2)}

In an argon-flushed two-neck round-bottom flask, 4,4,5,5-tetramethyl-2-(thiophen-2yl)1,3,2-dioxaborolane ( $0.146 \mathrm{~g}, 0.7 \mathrm{mmol})$ and 1,4-dibromo-2-ethoxy-5-methoxybenzene 
(0.093 g, $0.3 \mathrm{mmol})$ were dissolved in THF $(3 \mathrm{~mL})$ and degassed for $10 \mathrm{~min}$. Then tetrakis(triphenylphosphine)palladium $(0.048 \mathrm{~g}, 0.042 \mathrm{mmol})$ and $2 \mathrm{~mL}$ of the degassed solution of $\mathrm{Na}_{2} \mathrm{CO}_{3}(0.223 \mathrm{~g}, 2.1 \mathrm{mmol})$ in water were added. The mixture was heated at $70{ }^{\circ} \mathrm{C}$ and refluxed for $24 \mathrm{~h}$. After cooling down, the organic layer was extracted with $\mathrm{DCM}$ and dried over $\mathrm{MgSO}_{4}$. The solvent was removed with a rotary evaporator, and the residue was purified by column chromatography (eluent: hexane/ethyl acetate $=4 / 1$ ) to yield DTB2 as a yellow solid $(0.082 \mathrm{~g}, 0.26 \mathrm{mmol}, \mathrm{Y}=88 \%)$.

${ }^{1} \mathrm{H}$ NMR $\left(400 \mathrm{~Hz} ; \mathrm{CDCl}_{3}\right.$; TMS) $\delta(\mathrm{ppm}) 1.504-1.539(\mathrm{t}, 3 \mathrm{H}, J=7.0 \mathrm{~Hz}), 3.939(\mathrm{~s}$, $3 \mathrm{H}), 4.133-4.185(\mathrm{q}, 2 \mathrm{H}, J=6.9 \mathrm{~Hz}), 7.085-7.106(\mathrm{~m}, 2 \mathrm{H}), 7.241-7.261(\mathrm{~d}, 2 \mathrm{H}, J=8.0 \mathrm{~Hz})$, 7.326-7.340 (dd, $2 \mathrm{H}, J=4.0,5.6 \mathrm{~Hz}), 7.504-7.515(\mathrm{dd}, 1 \mathrm{H}, J=3.6,4.4 \mathrm{~Hz}), 7.549-7.558(\mathrm{~d}, 1 \mathrm{H}$, $J=3.6 \mathrm{~Hz}) .{ }^{13} \mathrm{C}$ NMR $(100 \mathrm{~Hz}$; CDCl 3 ; TMS) $\delta$ (ppm) 15.020, 56.413, 65.420, 86.903, 112.017, $113.427,122.920,123.187,125.198,125.408,125.627,125.722,126.742,126.875,139.066$, $139.199,149.178,149.921$.

\subsubsection{1,4-Dibromo-2-butoxy-5-methoxybenzene (12)}

2,5-Dibromo-4-methoxyphenol $(0.226 \mathrm{~g}, 0.8 \mathrm{mmol})$, tetrabutylammonium perchlorate $(0.01 \mathrm{~g}, 0.03 \mathrm{mmol})$, and potassium carbonate $(0.138 \mathrm{~g}, 1 \mathrm{mmol})$ were mixed in DMSO $(3 \mathrm{~mL})$. Bromobutane $(0.108 \mathrm{~mL}, 1 \mathrm{mmol})$ was added dropwise. Then, the mixture was stirred at room temperature for $24 \mathrm{~h}$. The mixture was extracted with DCM and dried over magnesium sulfate. Purification by silica gel column chromatography (eluent: hexane/ethyl acetate $=4 / 1)$ gave 12 as a yellow oil $(0.27 \mathrm{~g}, 0.8 \mathrm{mmol}, \mathrm{Y}=100 \%)$.

${ }^{1} \mathrm{H}$ NMR (400 MHz; CDCl 3 ; TMS) $\delta(\mathrm{ppm}) 0.964-1.001(\mathrm{t}, 3 \mathrm{H}, J=7.4 \mathrm{~Hz}), 1.474-1.568$ (sext, $2 \mathrm{H}, J=7.5 \mathrm{~Hz}$ ), 1.755-1.826 (quint, $2 \mathrm{H}, J=7.1 \mathrm{~Hz}$ ), $3.840(\mathrm{~s}, 3 \mathrm{H}), 3.942-3.974(\mathrm{t}, 2 \mathrm{H}$, $J=6.4 \mathrm{~Hz}), 7.087(\mathrm{~s}, 1 \mathrm{H}), 7.094(\mathrm{~s}, 1 \mathrm{H}) .{ }^{13} \mathrm{C} \mathrm{NMR}(100 \mathrm{MHz}$; CDCl $;$ TMS) $\delta$ (ppm) 13.829, $19.233,31.204,57.023,70.023,110.368,11.235,118.603,150.131,150.436$.

\subsubsection{1-Butoxy-4-methoxy-2,5-di(thiophen-2-yl)benzene (DTB4)}

In an argon-flushed two-neck round-bottom flask, 4,4,5,5-tetramethyl-2-(thiophen2-yl)1,3,2-dioxaborolane ( $0.210 \mathrm{~g}, 1 \mathrm{mmol})$ and 1,4-dibromo-2-butoxy-5-methoxybenzene $(0.169 \mathrm{~g}, 0.5 \mathrm{mmol})$ were dissolved in THF $(3 \mathrm{~mL})$ and degassed for $10 \mathrm{~min}$. Then, tetrakis(triphenylphosphine)palladium $(0.069 \mathrm{~g}, 0.06 \mathrm{mmol})$ and $2 \mathrm{~mL}$ of the degassed solution of $\mathrm{Na}_{2} \mathrm{CO}_{3}(0.318 \mathrm{~g}, 3 \mathrm{mmol})$ in water solution were added. The mixture was heated at $70{ }^{\circ} \mathrm{C}$ and refluxed for $24 \mathrm{~h}$. After cooling down, the organic layer was extracted with $\mathrm{DCM}$ and dried over $\mathrm{MgSO}_{4}$. The solvent was removed on a rotary evaporator, and the residue was purified by column chromatography (eluent: hexane/ethyl acetate $=4 / 1$ ) to yield 12 as a yellow solid $(0.129 \mathrm{~g}, 0.37 \mathrm{mmol}, \mathrm{Y}=75 \%)$.

${ }^{1} \mathrm{H}$ NMR $\left(400 \mathrm{~Hz} ; \mathrm{CDCl}_{3}\right.$; TMS) $\delta(\mathrm{ppm})$ 0.981-1.018 (t, 3H, $\left.J=7.4 \mathrm{~Hz}\right), 1.519-1.612$ (sext, 2H, $J=7.4 \mathrm{~Hz}$ ), 1.855-1.926 (quint, $2 \mathrm{H}, J=7.1 \mathrm{~Hz}$ ), $3.935(\mathrm{~s}, 3 \mathrm{H}), 4.073-4.106(\mathrm{t}, 2 \mathrm{H}$, $J=6.6 \mathrm{~Hz}), 7.081-7.103(\mathrm{dd}, 2 \mathrm{H}, J=3.6,5.2 \mathrm{~Hz}), 7.232-7.263(\mathrm{~d}, 2 \mathrm{H}, J=12.4 \mathrm{~Hz}), 7.328-7.341$ $(\mathrm{m}, 2 \mathrm{H}), 7.506-7.517(\mathrm{dd}, 1 \mathrm{H}, J=3.6,4.4 \mathrm{~Hz}), 7.532-7.545(\mathrm{~d}, 1 \mathrm{H}, J=1.6,3.6 \mathrm{~Hz}) .{ }^{13} \mathrm{C}$ NMR (100Hz; $\mathrm{CDCl}_{3}$; TMS) $\delta$ (ppm) 13.943, 19.500, 31.480, 56.480, 69.385, 112.112, 112.989, $122.920,123.006,125.160,125.389,125.617,125.722,126.704,126.866,139.123,139.189$, $149.340,149.778$.

\subsection{Electrochemical Polymerization in LCs}

The monomers and the supporting salt (TBAP) were added to a nematic LC medium and mixed thoroughly to obtain an LC electrolyte solution. An indium tin oxide (ITO)coated glass electrode was used as a substrate. For the electrochemical polymerization, the reaction cells were constructed using two ITO glass electrodes and a polytetrafluoroethylene spacer $(0.20 \mathrm{~mm}$ thickness) to construct a sandwich structure. The LC electrolyte solution was injected into the cell, heated to the isotropic phase, and gradually cooled to room temperature. The reaction cells were placed in a magnetic field (12 T, superconducting magnet) for $20 \mathrm{~min}$ in order to obtain the uniaxially oriented LC reaction medium. Then, $4.0 \mathrm{~V}$ of direct current was applied across the cell to initiate the polymerization at room 
temperature. After $15 \mathrm{~min}$, the power source was disconnected and the reaction cell was removed from the magnetic field. The resulting polymer film was washed with organic solvents to remove residual LC, supporting salt, and monomers. All the primary polymer films were reduced from an oxidized state to a neutral state with the treatment of hydrazine. The concentration of each component and the electrochemical polymerization conditions are summarized in Table 1.

Table 1. Concentration of each component of the electrolyte solution and the electrochemical polymerization conditions.

\begin{tabular}{lccc}
\hline \multicolumn{1}{c}{ Component } & Liquid Crystal & Supporting Salt & Reaction Units \\
\hline Molar concentration & $99 \%$ & $0.50 \%$ & $0.50 \%$ \\
Reaction time & & $15 \mathrm{~min}$ & \\
Voltage & & $4 \mathrm{~V}$ direct current & \\
Magnetic field intensity & $12 \mathrm{~T}$ \\
Temperature & rt. \\
Electrode substrate & ITO glass \\
\hline
\end{tabular}

\subsection{Characterization Methods}

${ }^{1} \mathrm{H}$ NMR and ${ }^{13} \mathrm{C}$ NMR spectra were recorded using a JNM-ECS spectrometer (JEOL, $400 \mathrm{MHz}$ ), the chemical shifts were recorded in parts per million, and the coupling constants $(J)$ were recorded in Hertz. POM observations were performed using a Nikon Eclipse LV100 POM with the crossed Nicol condition. LD UV-vis absorption spectroscopy was performed using a JASCO V-630 UV-vis spectrophotometer equipped with a polarizer.

\section{Results}

\subsection{Polymerization}

BT, d(BT), DTC, bis(DTC), DTB, bis(DTB), DTB2, and DTB4 as monomers were used in this study. BT, DTC, and DTB have one reactive unit (conjugated backbone) with two reactive sites ( $\alpha$-position of the thiophene unit) in each molecule, while $\mathbf{d}(\mathbf{B T})$, bis(DTC), and bis(DTB) have two independent reactive units with four reactive sites ( $\alpha$-position of the thiophene units) in each molecule. These characteristics allow $\mathbf{d}(\mathbf{B T})$, bis(DTC), and bis(DTB) to form a crosslinked polymer structure, and copolymerization with BT, DTC, and DTB can tune the degree of crosslinking. Compared with DTB, DTB2 and DTB4 have the same conjugated backbone but different alkyl side chain lengths. They were used for further investigation in the examination of molecular form influence. Plausible uncrosslinked and crosslinked forms are described in the polymerization of BT and $\mathrm{d}(\mathrm{BT})$, as an example (Figure 1).

BT is copolymerized with $\mathrm{d}(\mathrm{BT})$, DTC is copolymerized with dis(DTC), and DTB is copolymerized with bis(DTB). Hence, we distributed these six monomers into three copolymerization groups: the BT/d(BT) group, the $\mathbf{D T C} / \mathbf{b i s}(\mathrm{DTC})$ group, and the $\mathrm{DTB} / \mathbf{b i s}(\mathrm{DTB})$ group (Scheme 3). Given that the uncrosslinked monomer (uncrosslinkable) (BT, DTC, and DTB) has one reactive unit, while the crosslink monomer (crosslinkable) (d(BT), bis(DTC), and bis(DTB)) has two reactive units, the reactive unit concentration was used rather than monomer concentration for calculation in this work. For each polymerization, the total reactive unit concentration was kept at $0.5 \%$ molar. The ratio of crosslinked units (RCU) was adjusted as $0 \%, 30 \%, 50 \%, 70 \%$, and $100 \%$, in order to examine different degrees of crosslink. Herein, RCU is employed to briefly describe the proportion of added uncrosslinked units and crosslinked units, but it should be noted that it indicates no actual degree of crosslinking in each sample. 

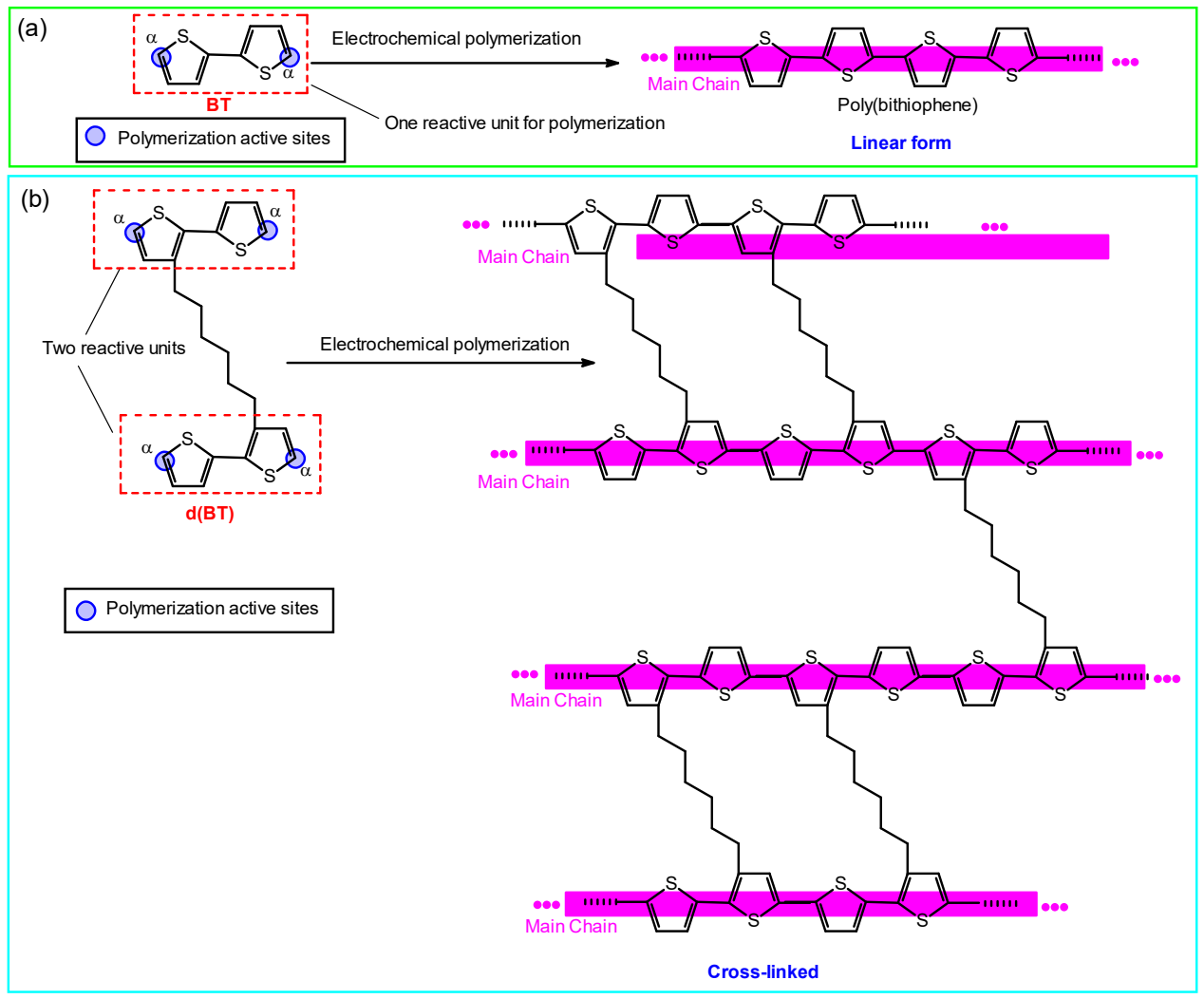

Figure 1. Plausible forms of linearly propagated BT ((a), uncrosslinkable) and $\mathbf{d}(\mathbf{B T})((\mathbf{b})$, crosslinkable).

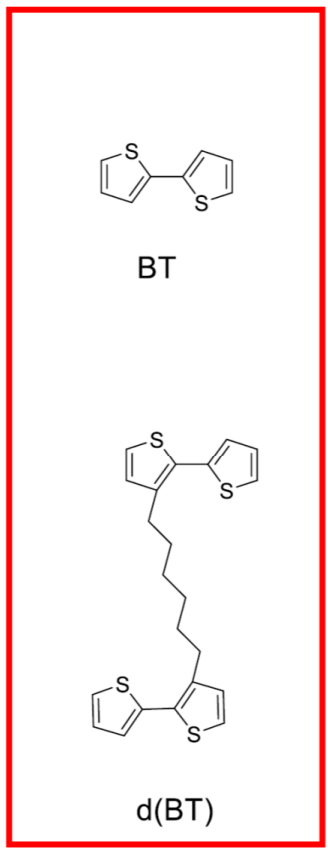

$\mathrm{BT} / \mathrm{d}(\mathrm{BT})$ group

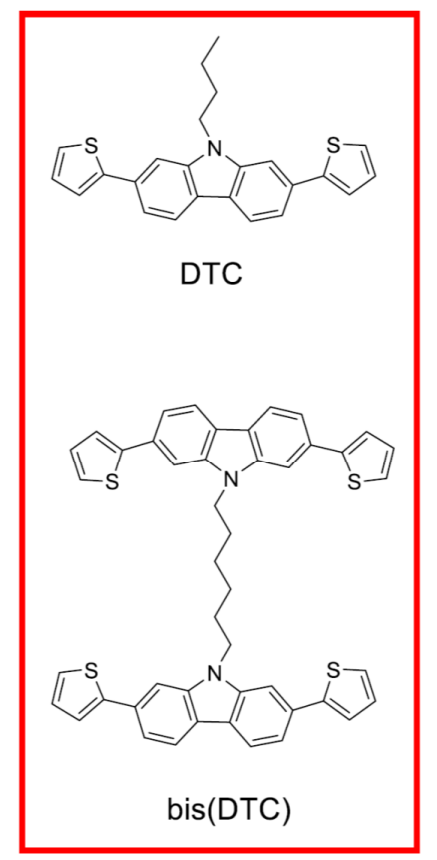

DTC/bis(DTC) group

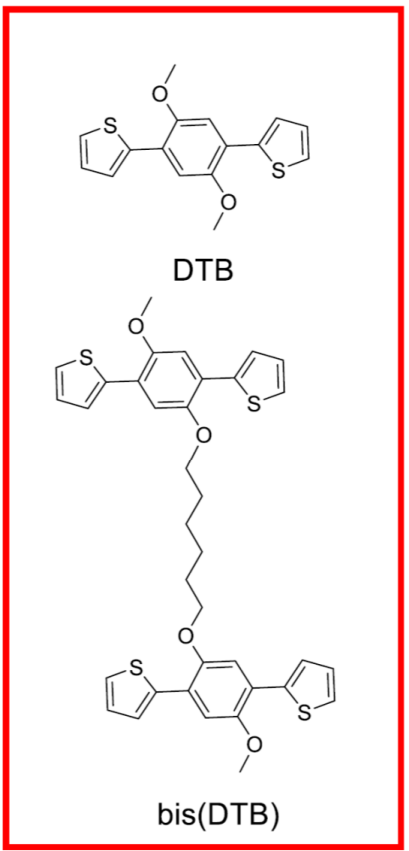

DTB/bis(DTB) group

Scheme 3. Six monomers are distributed between three groups in the experiments. The reactive unit concentration is used rather than the monomer concentration. In each electrolyte solution sample, the proportion of uncrosslinked units and crosslinked units is changed according to 10:0, 7:3, 5:5, 3:7, and 0:10 (in molar). 


\subsection{POM Observation}

Confirmation of birefringence is a convenient method to examine anisotropic materials. An anisotropic material possesses an optical axis. When linearly polarized light is applied to an anisotropic material, the electric vector of the light can be resolved into two vector components: one is parallel to the optical axis and the other is perpendicular to the optical axis. These two vector components of the light indicate the different propagation velocities in the material. Therefore, when these two vector components exit from the material, a phase difference between the optical waves is generated. Consequently, the electric vector of the new resulting polarized light can be differentiated from the original polarized light. This phenomenon can be described using the following equation:

$$
\Delta \varphi=2 \pi|n e-n o| / \lambda
$$

where $\Delta \varphi$ is the phase difference, $d$ is the thickness of the sample, $\lambda$ is the wavelength of the incident light, $n e$ is the refractive index of the polarized light, where the electric vector is parallel to the optical axis, and no is the refractive index of the polarized light, where the electric vector is perpendicular to the optical axis. When $\Delta \varphi=(2 n+1) \pi$, the direction of the electric vector of the emergent light is rotated $90^{\circ}$ from the incident light; when $\Delta \varphi=2 \mathrm{n} \pi$, the direction of the electric vector does not change; when $2 \mathrm{n} \pi<\Delta \varphi<(2 \mathrm{n}+1) \pi$, the emergent light becomes circularly polarized light or elliptically polarized light.

LC materials with no orientation usually show a characteristic optical texture, such as the Schlieren texture of nematic LCs (Figure 2A) and fan-shaped texture of smectic LCs (Figure 2B). These photographs show LC textures as examples.

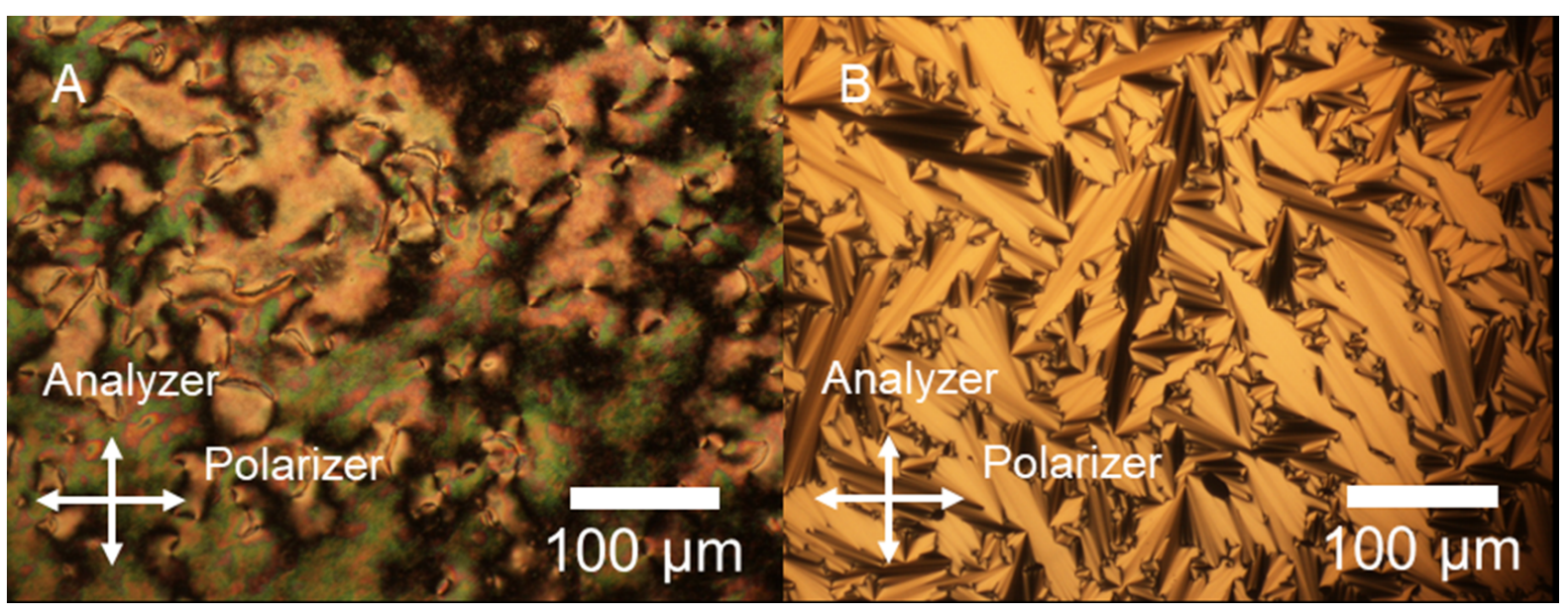

Figure 2. Polarized optical microscopy (POM) images of Schlieren texture of nematic liquid crystal LC (A) and fan-shaped texture of smectic LC (B).

Figure 3 shows the POM images of the polymer films obtained from the BT/d(BT) group, DTC/bis(DTC) group, and DTB/bis(DTB) group with varying RCU. The polymers were prepared in LC solution with electrochemical polymerization under the magnetic field. All samples displayed different colors and brightness levels between the left side and the right side of their pictures. When the LC orientation was parallel to the analyzer, dark pictures were observed, but when the LC orientation was not parallel to the analyzer and polarizer, significantly brighter pictures were observed. This difference was easily observed even at $100 \%$ RCU. Overall, this phenomenon indicates that all the samples were optically anisotropic, and their optical axes were parallel to the polarizer or analyzer. The conjugated polymers were uniaxially oriented even at $100 \%$ RCU. 


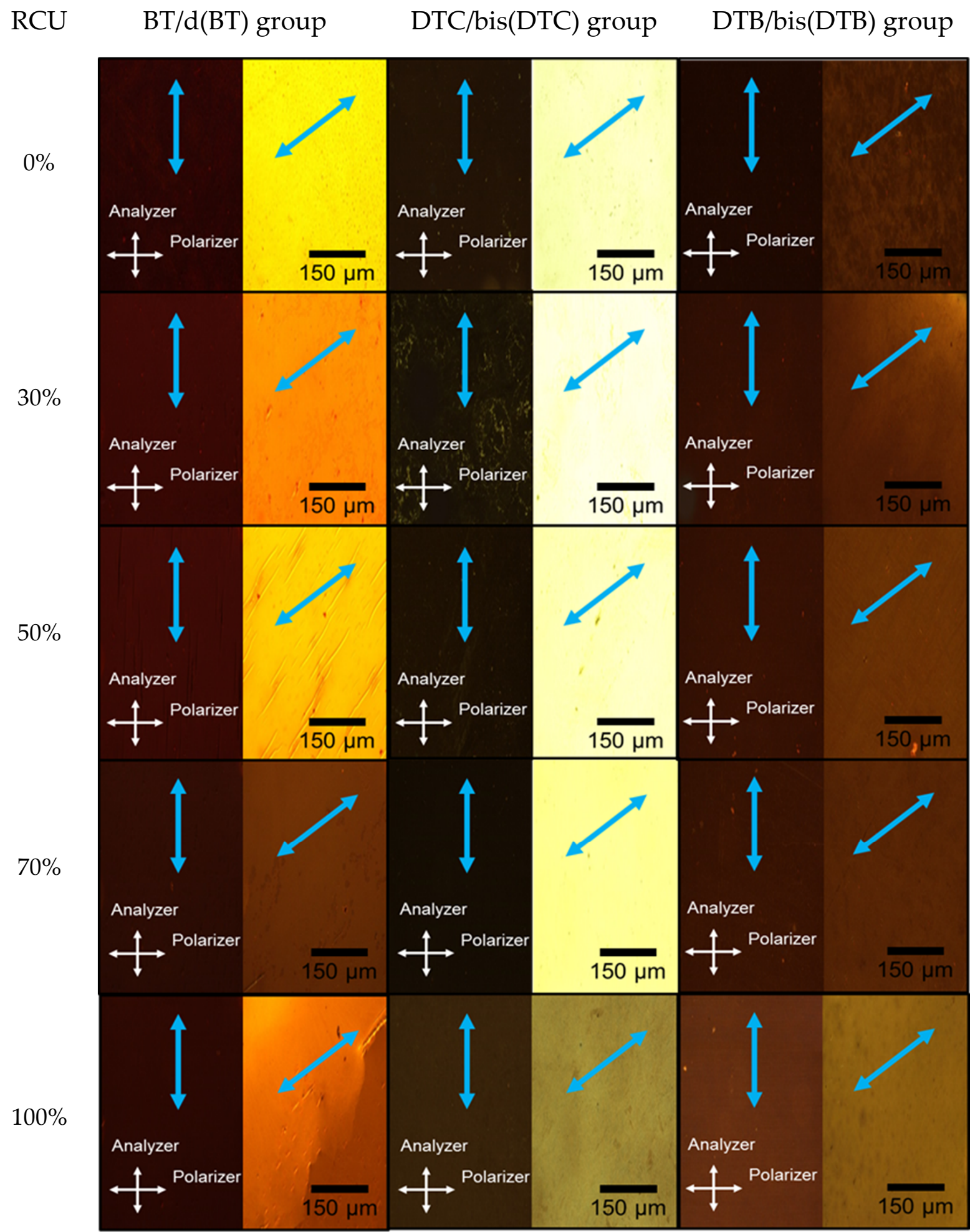

Figure 3. POM images of polymer films obtained from the BT/d(BT) group, DTC/bis(DTC) group, and DTB/bis(DTB) group with different RCUs. For comparison, a photograph was first taken of a sample (left side), and then the sample was rotated $45^{\circ}$ and a second photograph was taken (right side). Blue arrows indicate the orientation direction. RCU: Ratio of crosslinked units.

According to Equation (1), a low orientation degree will result in a small absolute value of $(n e-n o)$, which then results in a small phase difference $\Delta \varphi$. Therefore, it would be difficult to observe the emergent light using an analyzer, and changes in color or brightness 
between the left side and the right side of the POM image would also be imperceptible. In addition, the thickness of a sample $d$ can also affect $\Delta \varphi$. Therefore, the orientation degree of a polymer film cannot be evaluated only with the POM observation.

\subsection{LD UV-Vis Absorption Spectra Measurement}

In linearly conjugated polymers, the transition dipole moment is along the main chain. The electronic transition probability is along the direction proportional to the inner product between the dipolar transition moment and the electric vector of the incident light. If the polarization direction of the incident light is parallel to the main chain of a conjugated polymer, the maximum absorption intensity can be observed. Therefore, the main chain orientation of conjugated polymers can be defined using linear dichroism (LD) UV-vis absorption spectroscopy. The LD measurement for every sample was carried out two times in different orientations using a polarizer. One orientation was parallel to the LC orientation and the other was perpendicular to the LC orientation. If one of the two orientations shows a higher absorption intensity than the other, it indicates the main chain orientation of the polymers. Figure 4 shows the LD UV-vis spectra of all the samples. In each spectrum, the solid line represents the absorption intensity for an orientation that is parallel to the LC orientation, and the dashed line indicates the absorption intensity for an orientation that is perpendicular to the LC orientation. In the spectra of the $\mathbf{B T} / \mathbf{d}(\mathbf{B T})$ group and the DTC/bis(DTC) group, higher intensities were observed for the solid lines, indicating that the main chain orientation was parallel to the LC orientation. This is the parallel orientation. With an increase in the RCU, the intensity difference between the solid line and the dashed line decreased. This indicates that the increased proportion of crosslinked monomers resulted in a decrease in the orientation degree in this case.

In the LD spectra of the DTB/bis(DTB) group, when the RCU was $0 \%$, the dashed line showed a higher intensity, which indicates that the main chain orientation was perpendicular to the LC orientation. This is the perpendicular orientation. When the RCU was in the range of 30-70\%, the solid line and the dashed line had roughly the same intensity, which suggests that the orientation degree of the polymers was low. When the RCU was $100 \%$, the solid line gave a higher intensity, which means that the polymers were in a parallel orientation. For a better understanding of the effect of RCU on the orientation degree, a linear dichroism ratio (LDR) was summarized for all samples to evaluate the orientation degree. The LDR was calculated according to the following equation:

$$
\mathrm{LDR}=\mathrm{A}_{/ /} / \mathrm{A}_{\perp}
$$

where $A_{/ /}$is the maximum absorption intensity of the solid line and $A_{\perp}$ the maximum absorption intensity of the dashed line. When the LDR is greater than 1 , it indicates a parallel orientation; when the LDR is smaller than 1 , it indicates a perpendicular orientation; and when LDR is close to 1 , it indicates that the orientation degree decreased. The results are shown in Figure 5. It is worth noting that the addition of a crosslinked monomer changed both the orientation degree and the orientation direction of the polymers in the DTB/bis(DTB) group. Given that $100 \%$ DTB resulted in a perpendicular orientation and $100 \%$ bis(DTB) resulted in a parallel orientation, it follows that the blending of these two monomers would result in a neutralization of the parallel orientation effect and the perpendicular orientation effect. However, it is not clear what causes the different orientation directions of DTB and bis(DTB). It is likely that as DTB and bis(DTB) have the same conjugated backbone, the orientation difference arises due to the alkyl side chain and crosslinked structure. Therefore, the next step is to synthesize the monomers DTB2 (with an ethyl side chain) and DTB4 (with a butyl side chain), in order to investigate the effect of different alkyl side chains.

Figure 6 shows the LD UV-vis absorption spectra of the polymer films formed using the monomers DTB2 and DTB4. According to the spectra, DTB2 was in a perpendicular orientation while DTB4 was in a parallel orientation. This indicates that the orientation of conjugated polymers can be significantly affected with a slight change in the alkyl 
side chain length. It is clear that the addition of a crosslinked monomer dramatically affects the orientation degree of the polymers. In the BT/d(BT) and DTC/bis(DTC) groups, when the RCU increased to $30 \%$, the orientation degree significantly decreased; when the RCU was increased from $30 \%$ to $100 \%$, the orientation degree gradually decreased. In the DTB/bis(DTB) group, when the RCU was $0 \%$, the polymers showed a perpendicular orientation; when the RCU was $100 \%$, the polymers showed a parallel orientation; when the RCU was in the range of $30-70 \%$, the orientation degree was too small to define the orientation direction.

RCU

$\mathrm{BT} / \mathrm{d}(\mathrm{BT})$ group

DTC/bis(DTC) group

DTB/bis(DTB) group
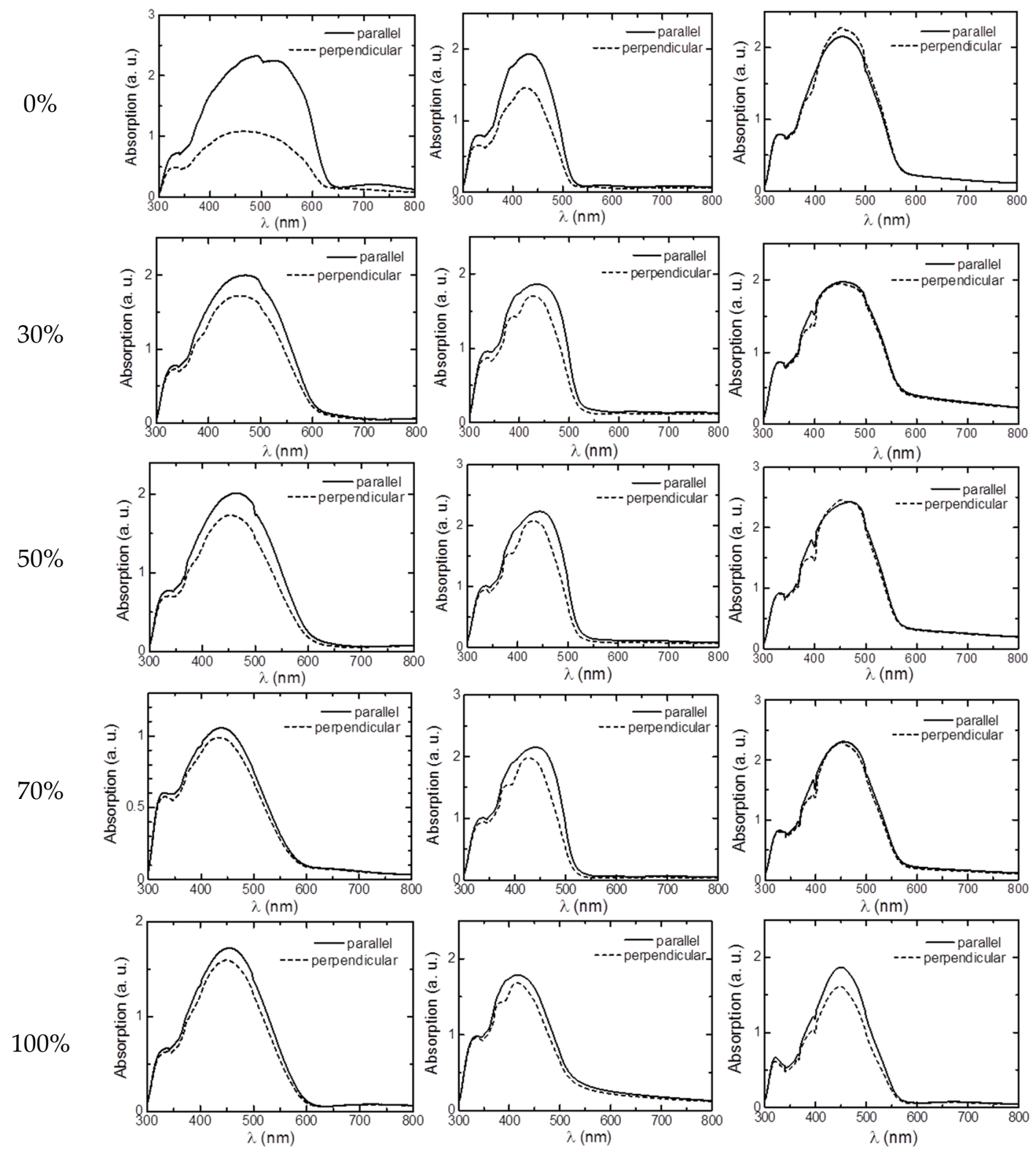

Figure 4. Linear dichroism (LD) UV-vis absorption spectra of polymer films obtained from the BT/d(BT) group, DTC/bis(DTC) group, and DTB/bis(DTB) group with different RCUs. In each spectrum, the solid line indicates the absorption intensity along the direction parallel to the liquid crystal orientation; the dashed line indicates the absorption intensity along the direction perpendicular to the liquid crystal orientation. LD: Linear dichroism. LDR: Linear dichroism ratio. RCU: Ratio of crosslinked units. 

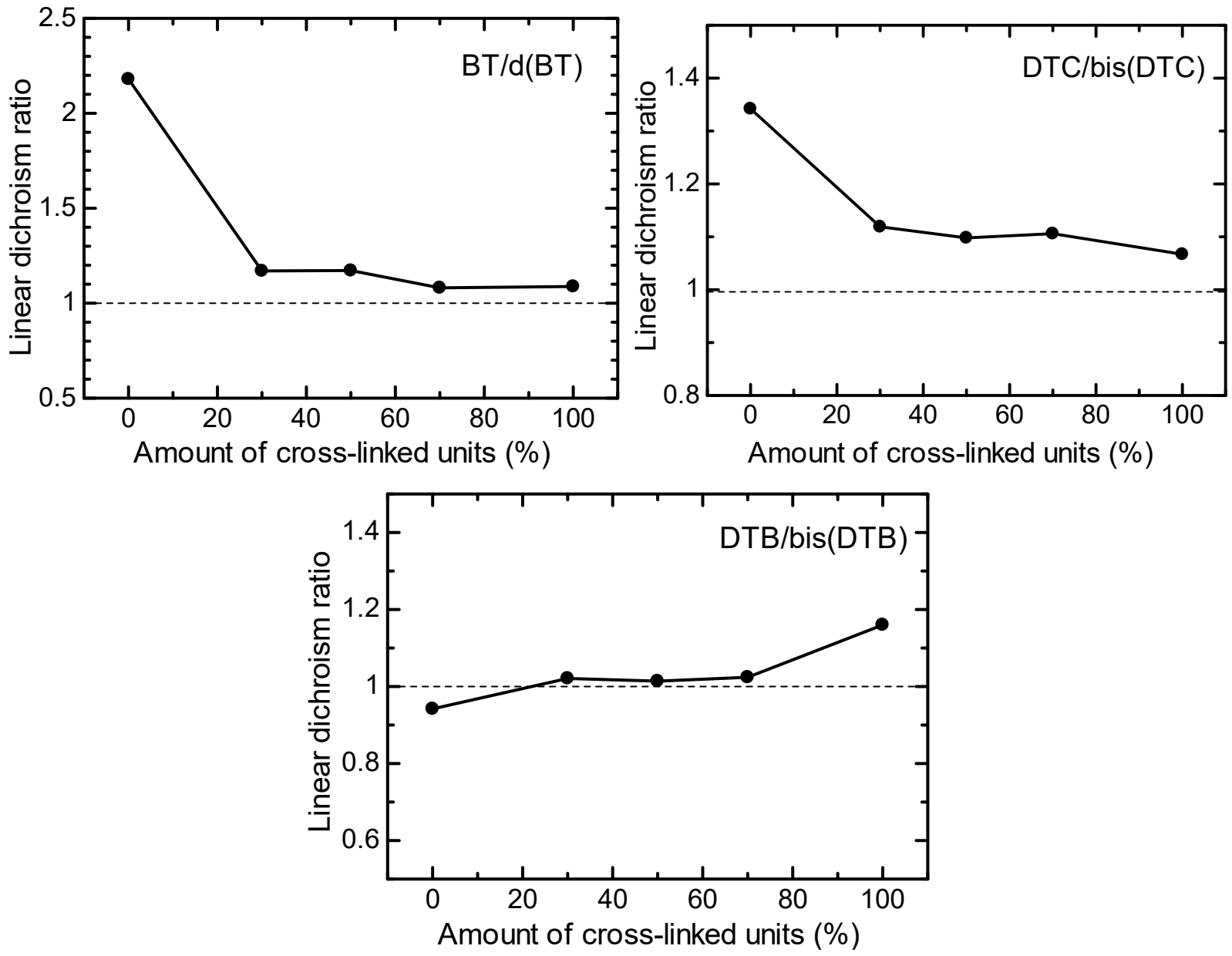

Figure 5. LDR of polymer films obtained from the BT/d(BT) group, DTC/bis(DTC) group, and DTB/bis(DTB) group with different RCUs. LDR: Linear dichroism ratio. RCU: Ratio of crosslinked units.
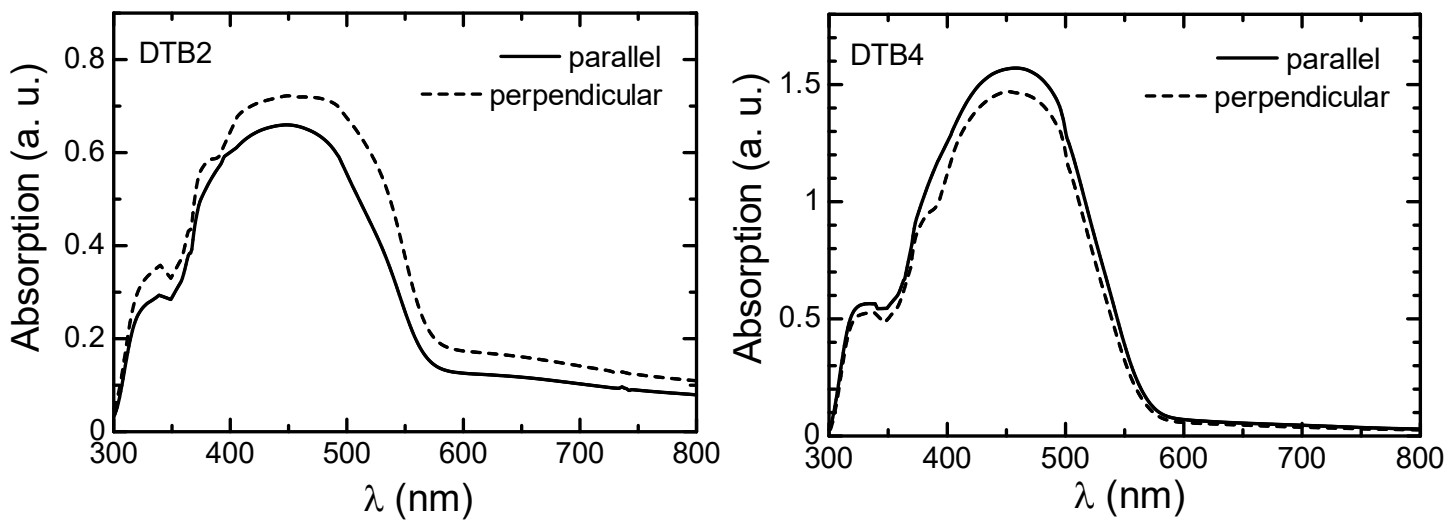

Figure 6. LD UV-vis absorption spectra of polymer films obtained from monomers DTB2 and DTB4. In each spectrum, the solid line indicates the absorption intensity along the direction parallel to the liquid crystal orientation; the dashed line indicates the absorption intensity along the direction perpendicular to the liquid crystal orientation.

\subsection{Optical Properties of Polymers}

Crosslinking affects not only the molecular orientation degree of polymers, but also their optical properties. The maximum absorption wavelength and the onset wavelength in the absorption spectra are two important parameters for evaluating the optical properties of a polymer. The maximum absorption wavelength and onset wavelength of the polymers are summarized in Figure 7. With a change in the RCU, the BT/d(BT), DTC/bis(DTC), 
and DTB/bis(DTB) groups showed completely different tendencies, which indicates the complexity of the crosslinking effect. For the BT/d(BT) group, an increased RCU resulted in a decrease in the onset and maximum absorption wavelengths; in the DTC/bis(DTC) group, the onset wavelength marginally changed while the maximum absorption wavelength first increased and then decreased; in the DTB/bis(DTB) group, the onset wavelength and maximum absorption wavelength changed in opposite directions. Given that the polymers formed within the same group have the same conjugated backbone structure, the change in the optical spectra may be due to three reasons: degree of polymerization, coplanarity of the conjugated backbone, or intermolecular interaction. When crosslinked monomers were added to the polymerization system at various concentrations, all three factors changed. It is most likely that the crosslinking effect changed the optical properties of the polymer films by affecting the molecular weight, coplanarity, and intermolecular interaction, simultaneously.
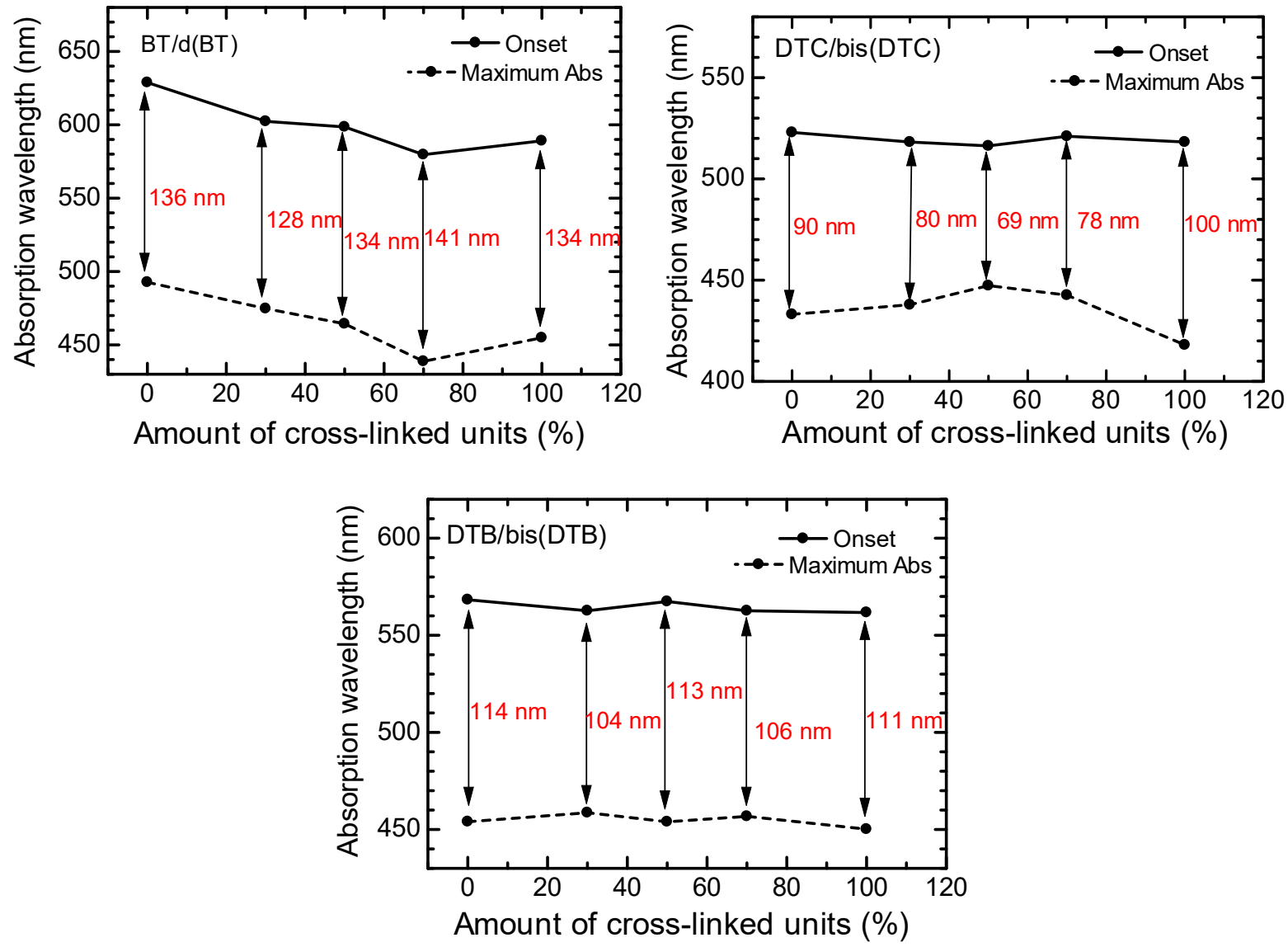

Figure 7. Maximum absorption wavelength and onset wavelength of polymer films synthesized from the BT/d(BT) group, DTC/bis(DTC) group, and DTB/bis(DTB) group with different RCUs. The differences between the maximum absorption wavelength and the onset wavelength are annotated in red letters.

Moreover, the difference between the two parameters was calculated. In general, the absorption spectra reflect the electronic transitions between the different vibrational energy levels of the ground and excited states. The onset wavelength corresponds to the $0-0$ transition and the maximum absorption wavelength corresponds to the transition with the highest transition probability. The difference between the onset wavelength and the maximum absorption wavelength can be reflected from mechanical twisting of the main chain in the excited state. 


\subsection{Solvent Resistance}

Most polymer films obtained via electrochemical polymerization in LCs are insoluble in organic solvents both in their oxidized state and after reduction (dedoped). However, the monomer DTB displays a unique property where the corresponding polymer film has solubility in THF after reduction with hydrazine. This property enables a closer examination of the effect of crosslinking on the solvent resistance. As shown in Figure 8, the poly(DTB) film was partly dissolved in THF. Poly(DTB) in THF solution was yellow, while poly(bis(DTB)) in THF solution was transparent, even though a small amount of yellow precipitate appeared. This contrast demonstrates that the crosslinking effect can improve the resistance to solvents in polymer films.

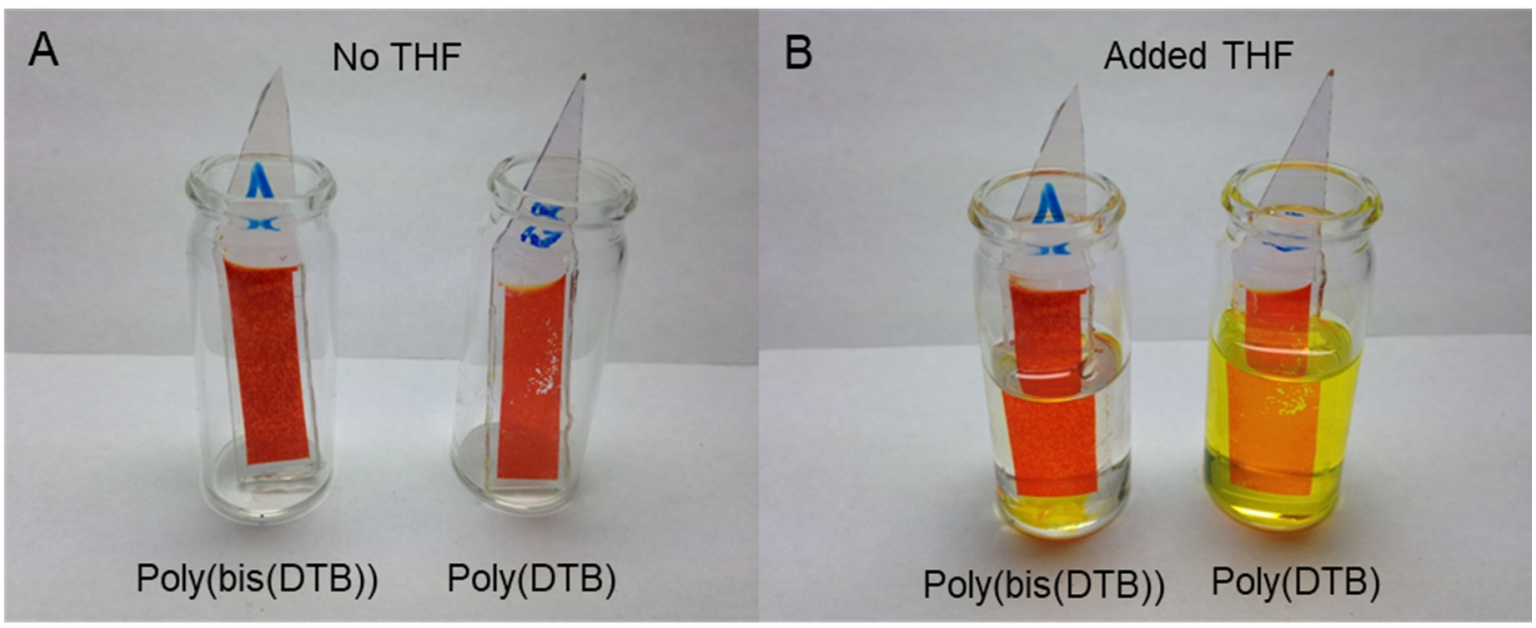

Figure 8. Solvent resistance of poly(DTB) and poly(bis(DTB)) to THF. (A) Before addition of THF; (B) after addition of THF.

\section{Conclusions}

In this research, a fabrication method for forming conjugated polymer films was developed using electrochemical polymerization in LCs. By employing the monomers with crosslink properties and magnetic orientation, the conjugated polymer films were fabricated with both a crosslinked network structure and a uniaxial main chain orientation. Two types of monomers, uncrosslinked monomers and crosslinked monomers, were employed for the electrochemical polymerization in LCs, and a magnetic orientation was performed to obtain a uniaxially oriented LC electrolyte solution for the polymerization. The resulting polymer films showed a uniform optical axis. This result indicates that the conjugated polymer network possesses a uniaxially oriented conjugated main chain. An increase in the proportion of crosslinked monomers resulted in a decrease in the orientation degree for the main chains in the polymer. Moreover, changes in the alkyl side chains length of the monomers induced a significant change in the orientation direction of the main chains in the resultant polymers. The crosslinking is beneficial in improving the durability for conjugated polymers.

\section{Instruments}

${ }^{1} \mathrm{H}$ NMR spectra were recorded on a JNM-ECS-400 NMR Spectrometer (JEOL, Tokyo, Japan). All signals were recorded in ppm with the internal TMS (tetramethylsilane) at $0.00 \mathrm{ppm}$. FT-IR absorption spectra were obtained with an FT/IR-4600 (JASCO, Tokyo, Japan) using the $\mathrm{KBr}$ method. All spectra were recorded between 400 and $4000 \mathrm{~cm}^{-1}$ with a resolution of $4 \mathrm{~cm}^{-1}$. POM observations were conducted with an Eclipse LV100 high-resolution polarizing microscope (Nikon, Tokyo, Japan). UV-vis absorption spectra were recorded on a V-630 UV-vis spectrophotometer (JASCO, Tokyo, Japan). 
Author Contributions: J.D. performed the synthesis of a series of the polymers, and spectroscopic measurements such as the IR, NMR, UV-vis, and POM observations. S.N. and J.D. carried out the magnetic orientations. J.D. and H.G. prepared the manuscript. H.G. conducted this project. All authors have read and agreed to the published version of the manuscript.

Funding: This research was supported by the Japan Society for the Promotion of Science (JSPS), Grants-in-Aid for Scientific Research (No. 20K05626).

Institutional Review Board Statement: Not applicable.

Informed Consent Statement: Not applicable.

Acknowledgments: We would like to thank the OPEN FACILITY, Research Facility Center for Science and Technology, University of Tsukuba, for allowing us to use the nuclear magnetic resonance spectrometer.

Conflicts of Interest: The authors declare no conflict of interest.

\section{References}

1. Horie, M.; Majewski, L.; Fearn, M.J.; Yu, C.-Y.; Luo, Y.; Song, A.; Saunders, B.R.; Turner, M. Cyclopentadithiophene based polymers-A comparison of optical, electrochemical and organic field-effect transistor characteristics. J. Mater. Chem. 2010, 20, 4347-4355. [CrossRef]

2. McCulloch, I.; Ashraf, R.S.; Biniek, L.; Bronstein, H.; Combe, C.; Donaghey, J.E.; James, D.I.; Nielsen, C.B.; Schroeder, B.C.; Zhang, W. Design of Semiconducting Indacenodithiophene Polymers for High Performance Transistors and Solar Cells. Acc. Chem. Res. 2012, 45, 714-722. [CrossRef] [PubMed]

3. Tanaka, H.; Watanabe, S.-I.; Ito, H.; Marumoto, K.; Kuroda, S.-I. Direct observation of the charge carrier concentration in organic field-effect transistors by electron spin resonance. Appl. Phys. Lett. 2009, 94, 103308. [CrossRef]

4. Guo, X.; Zhang, M.; Huo, L.; Xu, F.; Wua, Y.; Hou, J. Design, synthesis and photovoltaic properties of a new D- $\pi-A$ polymer with extended $\pi$-bridge units. J. Mater. Chem. 2012, 22, 21024-21031. [CrossRef]

5. Baek, N.S.; Hau, S.K.; Yip, H.-L.; Acton, O.; Chen, K.-S.; Jen, A. High Performance Amorphous Metallated $\pi$-Conjugated Polymers for Field-Effect Transistors and Polymer Solar Cells. Chem. Mater. 2008, 20, 5734-5736. [CrossRef]

6. Osken, I.; Gundogan, A.S.; Tekin, E.; Eroglu, M.S.; Ozturk, T. Fluorene-Dithienothiophene-S,S-dioxide copolymers. Fine-tuning for OLED applications. Macromolecules 2013, 46, 9202-9210. [CrossRef]

7. Zhao, Q.; Bai, Y.; Fan, Z.; Wang, P.; Li, J.; Li, D.; Zhang, X. Synthesis and characterization of a novel fluorene-alt-carbazole polymer to host green, blue, and red phosphorescence. J. Appl. Polym. Sci. 2015, 133. [CrossRef]

8. Ahn, T.; Lee, S.-G.; Shim, H.-K. Systematic approach of blue-light-emitting copolymers by introducing various naphthalene linkages into fluorene containing PPV derivatives. Opt. Mater. 2003, 21, 191-197. [CrossRef]

9. Gather, M.C.; Bradley, D.D.C. An improved optical method for determining the order parameter in thin oriented molecu-lar films and demonstration of a highly axial dipole moment for the lowest energy $\pi-\pi^{*}$ optical transition in poly(9,9-dioctylfluorene-cobithiophene). Adv. Funct. Mater. 2007, 17, 479-485. [CrossRef]

10. Dudenko, D.; Kiersnowski, A.; Shu, J.; Pisula, W.; Sebastiani, D.; Spiess, H.W.; Hansen, M.R. A Strategy for Revealing the Packing in Semicrystalline $\pi$-Conjugated Polymers: Crystal Structure of Bulk Poly-3-hexyl-thiophene (P3HT). Angew. Chem. 2012, 124, 11230-11234. [CrossRef]

11. King, S.; Vaughan, H.; Monkman, A. Orientation of triplet and singlet transition dipole moments in polyfluorene, studied by polarised spectroscopies. Chem. Phys. Lett. 2007, 440, 268-272. [CrossRef]

12. Kleinhenz, N.; Rosu, C.; Chatterjee, S.; Chang, M.; Nayani, K.; Xue, Z.; Kim, E.; Middlebrooks, J.; Russo, P.S.; Park, J.O.; et al. Liquid Crystalline Poly(3-hexylthiophene) Solutions Revisited: Role of Time-Dependent Self-Assembly. Chem. Mater. 2015, 27, 2687-2694. [CrossRef]

13. Oosterbaan, W.D.; Vrindts, V.; Berson, S.; Guillerez, S.; Douheret, O.; Ruttens, B.; D'Haen, J.; Adriaensens, P.; Manca, J.; Lutsen, L.; et al. Efficient formation, isolation and characterization of poly(3-alkylthiophene) nanofibres: Probing order as a function of side-chain length. J. Mater. Chem. 2009, 19, 5424-5435. [CrossRef]

14. Huang, Y.; Guo, X.; Liu, F.; Huo, L.; Chen, Y.; Russell, T.P.; Han, C.C.; Li, Y.; Hou, J. Improving the ordering and photovol-taic properties by extending $\pi$-conjugated area of electron-donating units in polymers with D-A structure. Adv. Mater. 2012, 24, 3383-3389. [CrossRef]

15. Koynov, K.; Bahtiar, A.; Ahn, T.; Cordeiro, R.M.; Ho1rhold, H.-H.; Bubeck, C. Molecular weight dependence of chain orien-tation and optical constants of thin films of the conjugated polymer MEH-PPV. Macromolecules 2006, 39, 8692-8698. [CrossRef]

16. Bae, J.W.; Song, K. Anisotropic charge-carrier mobilities of liquid crystalline conjugated polymers on photo-aligned PVCN dielectric insulators. Org. Electron. 2016, 30, 143-148. [CrossRef]

17. Yuan, K.; Chen, L.; Chen, Y. Photovoltaic performance enhancement of P3HT/PCBM solar cells driven by incorporation of conjugated liquid crystalline rod-coil block copolymers. J. Mater. Chem. C 2014, 2, 3835-3845. [CrossRef] 
18. Wang, A.; Kawabata, K.; Goto, H. Synthesis of isothianaphthene (ITN) and 3,4-ethylenedioxy-thiophene (EDOT)-based low-band gap liquid crystalline conjugated polymers. Materials 2013, 6, 2218-2228. [CrossRef]

19. Liu, C.; Tan, Y.; Li, C.; Wu, F.; Chen, L.; Chen, Y. Enhanced power-conversion efficiency in inverted bulk heterojunction so-lar cells using liquid-crystal-conjugated polyelectrolyte interlayer. ACS Appl. Mater. Int. 2015, 7, 19024-19033. [CrossRef] [PubMed]

20. Tong, X.; Han, D.; Fortin, D.; Zhao, Y. Highly Oriented Nanofibrils of Regioregular Poly(3-hexylthiophene) Formed via Block Copolymer Self-Assembly in Liquid Crystals. Adv. Funct. Mater. 2012, 23, 204-208. [CrossRef]

21. Moggio, I.; Moigne, J.L.; Arias-Marin, E.; Issautier, D.; Thierry, A.; Comoretto, D.; Dellepiane, G.; Cuniberti, C. Orientation of polydiacetylene and poly( $\pi$-phenylene ethynylene) films by epitaxy and rubbing. Macromolecules 2001, 34, 7091-7099. [CrossRef]

22. Kanbara, T.; Mori, C.; Wakayama, H.; Fukuda, T.; Zhou, Z.-H.; Maruyama, T.; Osakada, K.; Yamamoto, T. Orientation of linear $\pi$-conjugated poly( $\pi$-phenylene), poly(thiophene-2,5-diyl) and poly(2,2'-Bipyridine-5,5'-diyl) perpendicular to the surface of carbon and metal substrates. Factors controlling the orientation. Solid State Commun. 1992, 83, 771-774. [CrossRef]

23. Kim, N.-J.; Kwon, J.-H.; Kim, M. Highly Oriented Self-Assembly of Conducting Polymer Chains: Extended-Chain Crystallization during Long-Range Polymerization. J. Phys. Chem. C 2013, 117, 15402-15408. [CrossRef]

24. Yang, S.Y.; Shin, K.; Park, C.E. The Effect of Gate-Dielectric Surface Energy on Pentacene Morphology and Organic Field-Effect Transistor Characteristics. Adv. Funct. Mater. 2005, 15, 1806-1814. [CrossRef]

25. Kline, R.J.; McGehee, M.D. Morphology and Charge Transport in Conjugated Polymers. J. Macromol. Sci. Part C 2006, 46, 27-45. [CrossRef]

26. Davis, A.R.; Carter, K.R. Controlling Optoelectronic Behavior in Poly(fluorene) Networks Using Thiol-Ene Photo-Click Chemistry. Macromolecules 2015, 48, 1711-1722. [CrossRef]

27. Yang, Q.-D.; Cheng, Y.; Li, H.-W.; Liu, J.; Cheung, S.-H.; So, S.-K.; Wong, K.-W.; Lau, W.-M.; Tsang, S.-W. Locking the morphology with a green, fast and efficient physical cross-linking approach for organic electronic applications. Org. Electron. 2016, $28,53-58$. [CrossRef]

28. Clement, S.; Meyer, F.; Winter, J.D.; Coulembier, O.; Velde, C.M.L.V.; Zeller, M.; Gerbaux, P.; Balandier, J.-Y.; Sergeyev, S.; Lazzaroni, R.; et al. Synthesis and supramolecular organization of regioregular polythiophene block oligomers. J. Org. Chem. 2010, 75, 1561-1568. [CrossRef]

29. Wakabayashi, R.; Kaneko, K.; Takeuchi, M.; Shinkai, S. Toward the alignment of conjugated polymers into anisotropically-ordered structure. New J. Chem. 2007, 31, 790-799. [CrossRef]

30. Ling, X.; Pritzker, M.D.; Burns, C.M.; Byerley, J.J. A Mechanism for Electropolymerization of 2-Vinylpyridine Coatings on Metal Surfaces. Macromolecules 1998, 31, 9134-9140. [CrossRef]

31. Waltman, R.J.; Bargon, J. Electrically conducting polymers: A review of the electropolymerization reaction, of the effects of chemical structure on polymer film properties, and of applications towards technology. Can. J. Chem. 1986, 64, 76-95. [CrossRef]

32. Li, C.; Sun, C.; Chen, W.; Pan, L. Electrochemical thin film deposition of polypyrrole on different substrates. Surf. Coat. Technol. 2005, 198, 474-477. [CrossRef]

33. Liao, J.; Wu, S.; Yin, Z.; Huang, S.; Ning, C.; Tan, G.; Chu, P.K. Surface-Dependent Self-Assembly of Conducting Polypyrrole Nanotube Arrays in Template-Free Electrochemical Polymerization. ACS Appl. Mater. Interfaces 2014, 6, 10946-10951. [CrossRef] [PubMed]

34. Panasyuk, T.L.; Mirsky, V.; Piletsky, S.; Wolfbeis, O.S. Electropolymerized Molecularly Imprinted Polymers as Receptor Layers in Capacitive Chemical Sensors. Anal. Chem. 1999, 71, 4609-4613. [CrossRef]

35. Sharm, P.S.; Pietrzyk-Le, A.; D'Souza, F.; Kutner, W. Electrochemically synthesized polymers in molecular imprinting for chemical sensing. Anal. Bioanal. Chem. 2012, 402, 3177-3204. [CrossRef] [PubMed]

36. Carbas, B.B.; Kivrak, A.; Teke, E.; Zora, M.; Önal, A.M. Electrochemical polymerization of a new low-voltage oxidized thienylenepyrrole derivative and its electrochromic device application. J. Electroanal. Chem. 2014, 729, 15-20. [CrossRef]

37. Gu, C.; Dong, W.; Yao, L.; Lv, Y.; Zhang, Z.; Lu, D.; Ma, Y. Cross-Linked Multifunctional Conjugated Polymers Prepared by In Situ Electrochemical Deposition for a Highly-Efficient Blue-Emitting and Electron-Transport Layer. Adv. Mater. 2012, 24, $2413-2417$. [CrossRef] [PubMed]

38. Lv, Y.; Yao, L.; Gu, C.; Xu, Y.; Liu, D.; Lu, D.; Ma, Y. Electroactive self-assembled monolayers for enhanced efficiency and stabil-ity of electropolymerized luminescent films and devices. Adv. Funct. Mater. 2011, 21, 2896-2900. [CrossRef]

39. Yan, W.; Li, Y.; Sun, W.; Peng, H.; Ye, S.; Liu, Z.; Bian, Z.; Huang, C. High-performance hybrid perovskite solar cells with polythiophene as hole-transporting layer via electrochemical polymerization. RSC Adv. 2014, 4, 33039-33046. [CrossRef]

40. Goto, H.; Nimori, S. Liquid crystal electropolymerisation under magnetic field and resultant linear polarised electrochromism. J. Mater. Chem. 2010, 20, 1891-1898. [CrossRef]

41. Dong, J.; Kawabata, K.; Goto, H. Synthesis and characterization of a novel donor-acceptor-donor chiral inducer and its application in electrochemical polymerization. J. Mater. Chem. C 2015, 3, 2024-2032. [CrossRef]

42. Kawabata, K.; Takeguchi, M.; Goto, H. Optical activity of heteroaromatic conjugated polymer films prepared by asymmetric electrochemical polymerization in cholesteric liquid crystals: Structural function for chiral induction. Macromolecules 2013, 46, 2078-2091. [CrossRef]

43. Goto, H. Liquid crystal stepwise electropolymerization-an approach to create insect photonic structure. RSC Adv. 2013, 3, 6347-6355. [CrossRef] 
44. Yoneyama, H.; Tsujimoto, A.; Goto, H. Preparation of optically active pyridine-based conducting polymer films using a liquid crystal electrolyte containing a cholesterol derivative. Macromolecules 2007, 40, 5279-5283. [CrossRef]

45. Kawabata, K.; Nimori, S.; Goto, H. Horizontal and vertical orientation of polythiophenes by electrochemical polymerization in magnetically aligned smectic liquid crystal. ACS Macro Lett. 2013, 2, 587-591. [CrossRef]

46. Kawabata, K.; Goto, H. Electrosynthesis of 2,7-linked polycarbazole derivatives to realize low-band gap electroactive polymers. Synth. Met. 2010, 160, 2290-2298. [CrossRef] 